\title{
Dynamic Risk Measures and Path-Dependent Second Order PDEs
}

\author{
Jocelyne Bion-Nadal
}

\begin{abstract}
We propose new notions of regular solutions and viscosity solutions for path-dependent second order partial differential equations. Making use of the martingale problem approach to path-dependent diffusion processes, we explicitly construct families of time-consistent dynamic risk measures on the set of càdlàg paths $I R^{n}$ valued endowed with the Skorokhod topology. These risk measures are shown to have regularity properties. We prove then that these time-consistent dynamic risk measures provide viscosity supersolutions and viscosity subsolutions for path-dependent semi-linear second order partial differential equations.
\end{abstract}

Keywords Path-dependent PDE $\cdot$ Risk measures $\cdot$ Martingale problems

MSC: $35 \mathrm{D} 40 \cdot 35 \mathrm{R} 15 \cdot 35 \mathrm{~K} 55 \cdot 60 \mathrm{~J} 60 \cdot 91 \mathrm{~B} 30$

\section{Introduction}

Diffusion processes are linked with parabolic second order Partial Differential Equations via the "Feynman-Kac" formula. The field of path-dependent PDEs first started in 2010 when Peng asked in [19] whether a BSDE (Backward Stochastic Differential Equation first introduced in [17]) could be considered as a solution to a path-dependent PDE. In line with the recent literature on the topic, a solution to a path-dependent second order PDE

$$
H\left(u, \omega, \phi(u, \omega), \partial_{u} \phi(u, \omega), D_{x} \phi(u, \omega), D_{x}^{2} \phi(u, \omega)\right)=0
$$

is searched as a progressive function $\phi(u, \omega)$ (i.e. a path dependent function depending at time $u$ on all the path up to time $u$ ).

J. Bion-Nadal $(\varangle)$

CMAP (UMR CNRS 7641), Ecole Polytechnique, 91128 Palaiseau Cedex, France

e-mail: jocelyne.bion-nadal@cmap.polytechnique.fr 
In contrast with the classical setting, the notion of regular solution for a pathdependent PDE (1) needs to deal with càdlàg paths. Indeed to give a meaning to the partial derivatives $D_{x} \phi(u, \omega)$ and $D_{x}^{2} \phi(u, \omega)$ at $\left(u_{0}, \omega_{0}\right)$, one needs to assume that $\phi\left(u_{0}, \omega\right)$ is defined for paths $\omega$ admitting a jump at time $u_{0}$. Peng has introduced in [20] a notions of regular and viscosity solution for path-dependent second order PDEs. In [20] a regular or a viscosity solution for a path-dependent PDE is a progressive function $\phi(t, \omega)$ defined on the space of càdlàg paths endowed with the uniform norm topology and the notion of continuity and partial derivatives are those introduced by Dupire [12]. A comparison theorem is proved in this setting [20]. The motivation comes mainly from the theory of BSDE and examples of regular solutions to path-dependent PDEs can be constructed from BSDEs [18]. The main drawback for this approach based on [12] is that the uniform norm topology on the set of càdlàg paths is not separable, hence it is not a Polish space. Recently Ekren et al. proposed a notion of viscosity solution for path-dependent PDEs in the setting of continuous paths in $[13,14]$. This work was motivated by the fact that a continuous function defined on the set of continuous paths does not have a unique extension into a continuous function on the set of càdlàg paths. Therefore it is suitable that the notion of viscosity solution for functions defined only on the set of continuous paths does not require to extend the function to the set of càdlàg paths. The approach developed in $[13,14]$ is also based on BSDE.

In the present paper we introduce a new notion of regular and viscosity solution for path-dependent second order PDEs (Sect.2). A solution to (1) is a progressive function $\phi$ defined on $R_{+} \times \Omega$ where $\Omega$ is the set of càdlàg paths. In contrast with [20] and many works on path-dependent problems, we consider the Skorokhod topology on the set of càdlàg paths. Thus $\Omega$ is a Polish space. This property is very important. To define the continuity and regularity properties for a progressive function, we make use of the one to one correspondence between progressive functions on $R_{+} \times \Omega$ and strictly progressive functions on $\mathbb{R}_{+} \times \Omega \times \mathbb{R}^{n}$ established in [3]. A function $\phi$ defined on $\mathbb{R}_{+} \times \Omega$ is progressive if $\phi(s, \omega)=\phi\left(s, \omega^{\prime}\right)$ as soon as $\omega(u)=\omega^{\prime}(u)$ for all $0 \leq u \leq s$. A function $\bar{\phi}$ defined on $\mathbb{R}_{+} \times \Omega \times \mathbb{R}^{n}$ is strictly progressive if $\bar{\phi}(s, \omega, x)=\bar{\phi}\left(s, \omega^{\prime}, x\right)$ as soon as $\omega(u)=\omega^{\prime}(u)$ for all $0 \leq u<s$. The one to one correspondence is given by $\bar{\phi}(s, \omega, x)=\phi\left(s, \omega *_{s} x\right)$ where $\left(\omega *_{s} x\right)(u)=\omega(u)$ for all $0 \leq u<s$ and $\left(\omega *_{s} x\right)(u)=x$ for all $u \geq s$. The continuity and regularity properties that we want for a progressive function $\phi$ are derived from the usual continuity and regularity properties for $\bar{\phi}$ via the above one to one correspondence. For example, $D_{x} \phi(u, \omega)$ is defined as $D_{x} \phi(u, \omega):=D_{x} \bar{\phi}(u, \omega, \omega(u))$ where $D_{x} \bar{\phi}$ is the usual partial derivative of $\bar{\phi}$ with respect to the third variable. Notice that via the above one to one correspondence, the regularity properties for a progressive function $\phi$ are defined in a very natural way. This is in contrast with the most commonly used regularity definitions first introduced in [12].

The notion of viscosity solution that we introduce in the present paper is motivated by our construction of a solution to semi-linear second order path-dependent PDEs based on the martingale problem approach.

Our study for viscosity solutions of path-dependent PDEs allows then to introduce a new definition of viscosity solution for path-dependent functions defined only on the 
set of continuous paths. As in $[13,14]$, this does not require to extend the function nor the coefficient functions appearing in the path-dependent PDE to the set of càdlàg paths. However our approach is very different from the one introduced in $[13,14]$. In the present paper we construct then time consistent dynamic risk measures on the set $\Omega$ of càdlàg paths, to produce solutions for path-dependent semi-linear second order PDEs.

$$
\left\{\begin{aligned}
\partial_{u} v(u, \omega)+\mathscr{L}^{a} v(u, \omega)+f\left(t, \omega, D_{x} v(u, \omega)\right) & =0 \text { on }[0, t] \times \Omega \\
v(t, \omega) & =h(\omega)
\end{aligned}\right.
$$

with $\mathscr{L}^{a} v(u, \omega)=\frac{1}{2} \operatorname{Tr}\left[a(u, \omega) D_{x}^{2} v(u, \omega)\right]$.

These dynamic risk measures are constructed using probability measures solution to a path-dependent martingale problem. This approach is motivated by the Feynman Kac formula and more specifically by the link between solutions of a parabolic second order PDE and probability measures solutions to a martingale problem. The martingale problem has been first introduced and studied by Stroock and Varadhan $[10,11]$ in the case of continuous diffusion processes. The martingale problem is linked to stochastic differential equations. However the martingale problem formulation is intrinsic and is very well suitable to construct risk measures. In [22] the martingale problem has been extended and studied to the case of jump diffusions. In [3], the study of the martingale problem is extended to the path-dependent case which means that the functions $a$ and $b$ (and also the jump measure) are no more defined on $I R_{+} \times \mathbb{R}^{n}$ but on $I R_{+} \times \Omega$. The question of existence and uniqueness of a solution to a path-dependent martingale problem is addressed in [3] in a general setting of diffusions with a path-dependent jump term. In the case where there is no jump term and under Lipschitz conditions on the coefficients, the existence and uniqueness of a solution has been already established in [8] from the stochastic differential equation point of view.

In Sect.3, we recall some results from [3] on the martingale problem for pathdependent diffusion processes and study the support of a probability measure solution to the path-dependent martingale problem for $\mathscr{L}^{a, b}$.

The theory of dynamic risk measures on a filtered probability space has been developped in recent years. In the case of a Brownian filtration, dynamic risk measures coincide with $g$-expectations introduced by Peng [21]. An important property for dynamic risk measures is time consistency. The time consistency property for dynamic risk measures is the analogue of the Dynamic Programming Principle. For sublinear dynamic risk measures time consistency has been characterized by Delbaen [9]. For general convex dynamic risk measures two different characterizations of time consistency have been given. One by Cheridito et al. [7], the other by BionNadal [5]. This last characterization of time consistency is very useful in order to construct time consistent dynamic risk measures.

Following [5], one can construct a time consistent dynamic risk measure as soon as one has a stable set of equivalent probability measures $\mathscr{Q}$ and a penalty defined on $\mathscr{Q}$ satisfying some conditions. In Sect. 4 , we construct a stable set of probability measures on the set $\Omega$ of càdlàg paths. In the whole paper $a$ is a given bounded 
progressively continuous function defined on $I R_{+} \times \Omega$ such that $a(s, \omega)$ is invertible for all $(s, \omega)$. For all $r \geq 0$ and $\omega \in \Omega$ the set of probability measures $\mathscr{Q}_{r, \omega}$ is a stable set generated by probability measures $Q_{r, \omega}^{a, b}$ solution to the martingale problem for $\mathscr{L}^{a, b}$ starting from $\omega$ at time $r$. The functions $b$ are assumed to satisfy some uniform $B M O$ condition. In Sect. 5 we construct penalties on the stable set $\mathscr{Q}_{r, \omega}$ from a pathdependent function $g$. Some growth conditions are assumed on the function $g$ to ensure integrability properties for the penalties. With such a stable set and penalties, we construct in Sect. 6 time consistent convex dynamic risk measures. More precisely for all $r$ and $\omega$ we construct a time consistent convex dynamic risk measure $\rho_{s, t}^{r, \omega}$ on the filtered probability space $\left(\Omega,\left(\mathscr{B}_{t}\right), Q_{r, \omega}^{a}\right)$ where $Q_{r, \omega}^{a}$ means $Q_{r, \omega}^{a, 0}$ and $\left(\mathscr{B}_{t}\right)$ is the canonical filtration.

We prove furthermore in Sect. 7 that these time consistent dynamic risk measures satisfy the following Feller property: Let $\mathscr{C}_{t}$ be the set of $\mathscr{B}_{t}$ measurable functions $h$ defined on $\Omega$ which can be written as $h(\omega)=k(\omega, \omega(t))$ for some continuous function $k$ on $\Omega \times \mathbb{R}^{n}$ such that $k(\omega, x)=k\left(\omega^{\prime}, x\right)$ if $\omega(u)=\omega^{\prime}(u)$ for all $u<t$. Then for all $h$ in $\mathscr{C}_{t}$, there is a progressively lower semicontinuous function $R(h)$ on $[0, t] \times \Omega$ such that $R(h)(t, \omega)=h(\omega)$,

$$
\rho_{r, t}^{r, \omega_{0}}(h)=R(h)\left(r, \omega_{0}\right) \forall 0 \leq r \leq t
$$

Furthermore, for all $0 \leq r \leq s \leq t$,

$$
\rho_{s, t}^{r, \omega_{0}}(h)\left(\omega^{\prime}\right)=R(h)\left(s, \omega^{\prime}\right) Q_{r, \omega_{0}}^{a} \text { a.s. }
$$

We prove furthermore in Sect. 8 that the lower semicontinuous function $R(h)$ is a viscosity supersolution for the path-dependent semi linear second order partial differential equation (2). The function $f: \mathbb{R}^{+} \times \mathbb{R}^{n} \times \mathbb{R}^{d} \rightarrow \mathbb{R}$ appearing in Eq. (2) is linked to the choice of the penalty of the risk measure. It is convex in the last variable.

We prove also that the upper semi-continuous envelope of $R(h)$ is a viscosity subsolution for (2).

When the above function $h$ is defined only on the set of continuous paths, it is the same for the function $R(h)$. We prove then that $R(h)$ provides a viscosity supersolution and a viscosity subsolution for (2) on the set of continuous paths.

\section{Solution of Path-dependent PDEs}

In this section we introduce new notions for regular and viscosity solutions for second order path-dependent PDEs on the set of càdlàg paths. In contrast with [20] and all the papers using the notions of continuity and derivative introduced by Dupire [12], we work with the Skorohod topology on the set of càdlàg paths. A solution to a path-dependent PDE (1) is a progressive function $\phi(t, \omega)$ where $t$ belongs to $\mathbb{R}_{+}$and $\omega$ belongs to the set of càdlàg paths. 


\subsection{Topology and Regularity Properties}

In the whole paper $\Omega$ denotes the set of càdlàg paths with the Skorohod topology. The set $\Omega$ is then a Polish space (i.e. is metrizable and separable). Polish spaces have nice properties which are very important in the construction of solutions for pathdependent PDEs. Among them are the existence of regular conditional probability distributions, the equivalence between relative compactness and tightness for a set of probability measures, to name a few.

To define the continuity and regularity properties for progressive functions, we use the one to one correspondence between progressive functions on $I R_{+} \times \Omega$ and strictly progressive functions on $\mathbb{R}_{+} \times \Omega \times \mathbb{R}^{n}$ that we have established in [3].

A function $\phi$ defined on $\mathbb{R}_{+} \times \Omega$ is progressive if $\phi(s, \omega)=\phi\left(s, \omega^{\prime}\right)$ as soon as $\omega(u)=\omega^{\prime}(u)$ for all $0 \leq u \leq s$.

A function $\bar{\phi}$ defined on $\mathbb{R}_{+} \times \Omega \times \mathbb{R}^{n}$ is strictly progressive if $\bar{\phi}(s, \omega, x)=$ $\bar{\phi}\left(s, \omega^{\prime}, x\right)$ as soon as $\omega(u)=\omega^{\prime}(u)$ for all $0 \leq u<s$.

The one to one correspondence $\phi \rightarrow \bar{\phi}$ is given by $\bar{\phi}(s, \omega, x)=\phi\left(s, \omega *_{s} x\right)$ where

$$
\omega *_{s} x(u)=\omega(u) \forall 0 \leq u<s \text { and } \omega *_{s} x(u)=x \forall u \geq s .
$$

Notice that $\phi(s, \omega)=\bar{\phi}(s, \omega, \omega(s))$. Accordingly a progressive function $\phi$ (in 2 variables $(s, \omega))$ is said to be progressively continuous if the associated function $\bar{\phi}$ (in 3 variables $(s, \omega, x)$ ) is continuous on $\mathbb{R}_{+} \times \Omega \times \mathbb{R}^{n}$.

\subsection{Regular Solution}

Making use of the one to one corrrespondence between progressive functions on $R_{+} \times \Omega$ and strictly progressive functions on $I R_{+} \times \Omega \times \mathbb{R}{ }^{n}$, we can then give the following definition for a solution to a general path-dependent PDE.

Definition 1 Let $v$ be a progressive function on $I R_{+} \times \Omega$ where $\Omega$ is the set of càdlàg paths with the Skorokhod topology. $v$ is a regular solution to the following path-dependent second order PDE

$$
H\left(u, \omega, v(u, \omega), \partial_{u} v(u, \omega), D_{x} v(u, \omega), D_{x}^{2} v(u, \omega)\right)=0
$$

if the function $\bar{v}$ belongs to $\mathscr{C}^{1,0,2}\left(\mathbb{R}_{+} \times \Omega \times \mathbb{R}^{n}\right)$ and if the usual partial derivatives of $\bar{v}$ satisfy the equation

$$
H\left(u, \omega *_{u} x, \bar{v}(u, \omega, x), \partial_{u} \bar{v}(u, \omega, x), D_{x} \bar{v}(u, \omega, x), D_{x}^{2} \bar{v}(u, \omega, x)=0\right.
$$

with $\bar{v}(u, \omega, x)=v\left(u, \omega *_{u} x\right)$ 
$\left(\omega *_{u} x\right)(s)=\omega(s) \forall s<u$, and $\left(\omega *_{u} x\right)(s)=x \forall s \geq u$. The partial derivatives of $\bar{v}$ are the usual ones, the continuity notion for $\bar{v}$ is the usual one.

\subsection{Viscosity Solutions on the Set of Càdlàg Paths}

The following definitions are motivated by the construction of viscosity solutions for path-dependent PDEs that we develop in the following sections. Our construction of solutions is based on the martingale problem approach for path-dependent diffusions. The support of every probability measure $Q_{r, \omega_{0}}^{a, b}$ solution to the martingale problem for $\mathscr{L}^{a, b}$ starting from $\omega_{0}$ at time $r$ is contained in the set of paths which coincide with $\omega_{0}$ up to time $r$. This is a motivation for the following weak notion of continuity and also for the weak notion of local minimizer (or local maximizer) that we introduce in the definition of viscosity solution.

Definition 2 A progressively measurable function $v$ defined on $I R_{+} \times \Omega$ is continuous in viscosity sense at $\left(r, \omega_{0}\right)$ if

$$
v\left(r, \omega_{0}\right)=\lim _{\varepsilon \rightarrow 0}\left\{v(s, \omega),(s, \omega) \in D_{\varepsilon}\left(r, \omega_{0}\right)\right\}
$$

where

$$
\begin{array}{r}
D_{\varepsilon}\left(r, \omega_{0}\right)=\left\{(s, \omega), r \leq s<r+\varepsilon, \omega(u)=\omega_{0}(u), \forall 0 \leq u \leq r\right. \\
\left.\omega(u)=\omega(s) \forall u \geq s, \text { and } \sup _{r \leq u \leq s}\left\|\omega(u)-\omega_{0}(r)\right\|<\varepsilon\right\}
\end{array}
$$

$v$ is lower (resp. upper) semi continuous in viscosity sense if Eq. (6) is satisfied replacing lim by lim inf (resp. lim sup).

Definition 3 Let $v$ be a progressively measurable function on $\left(\mathbb{R}_{+} \times \Omega,\left(\mathscr{B}_{t}\right)\right)$ where $\Omega$ is the set of càdlàg paths with the Skorokhod topology and $\left(\mathscr{B}_{t}\right)$ the canonical filtration.

1. $v$ is a viscosity supersolution of (4) if $v$ is lower semi-continuous in viscosity sense, and if for all $\left(t_{0}, \omega_{0}\right) \in \mathbb{R}_{+} \times \Omega$,

- $v$ is bounded from below on $D_{\varepsilon}\left(t_{0}, \omega_{0}\right)$ for some $\varepsilon>0$.

- for all strictly progressive function $\bar{\phi} \in \mathscr{C}_{b}^{1,0,2}\left(\mathbb{R}_{+} \times \Omega \times \mathbb{R}^{n}\right)$ such that $v\left(t_{0}, \omega_{0}\right)=\phi\left(t_{0}, \omega_{0}\right)$, and $\left(t_{0}, \omega_{0}\right)$ is a minimizer of $v-\phi$ on $D_{\varepsilon}\left(t_{0}, \omega_{0}\right)$ for some $\varepsilon>0$,

$$
H\left(u, \omega *_{u} x, \phi(u, \omega, x), \partial_{u} \phi(u, \omega, x), D_{x} \phi(u, \omega, x), D_{x}^{2} \phi(u, \omega, x) \geq 0\right.
$$

at point $\left(t_{0}, \omega_{0}, \omega_{0}\left(t_{0}\right)\right)$. 
2. $v$ is a viscosity subsolution of (4) if $v$ is upper semi-continuous in viscosity sense, and for all $\left(t_{0}, \omega_{0}\right)$,

- $v$ is bounded from above on $D_{\varepsilon}\left(t_{0}, \omega_{0}\right)$ for some $\varepsilon>0$

- for all strictly progressive function $\bar{\phi} \in \mathscr{C}_{b}^{1,0,2}\left(\mathbb{R}_{+} \times \Omega \times \mathbb{R}^{n}\right)$ such that $v\left(t_{0}, \omega_{0}\right)=\phi\left(t_{0}, \omega_{0}\right)$, and $\left(t_{0}, \omega_{0}\right)$ is a maximizer of $v-\phi$ on $D_{\varepsilon}\left(t_{0}, \omega_{0}\right)$ for some $\varepsilon>0$,

$$
H\left(u, \omega *_{u} x, \phi(u, \omega, x), \partial_{u} \phi(u, \omega, x), D_{x} \phi(u, \omega, x), D_{x}^{2} \phi(u, \omega, x) \leq 0\right.
$$

at point $\left(t_{0}, \omega_{0}, \omega_{0}\left(t_{0}\right)\right)$.

3. $v$ is a viscosity solution if $v$ is both a viscosity supersolution and a viscosity subsolution.

\subsection{Viscosity Solution on the Set of Continuous Paths}

Recently Ekren et al. [13, 14] introduced a notion of viscosity solution of a pathdependent second order PDE for a function $v$ defined on the set of continuous paths. One motivation for this was to define the notion of viscosity solution without extending the function $v$ to the set of càdlàg paths.

We can notice that within our setting we can also define a notion of viscosity solution for a function $v$ defined only on the set of continuous paths without extending $v$. We give the following definition which is very different from that of $[13,14]$ and much simpler.

Definition 4 Let $v$ be a progressively measurable function defined on $I R_{+} \times$ $\mathscr{C}\left(R_{+}, I^{n}\right)$ with the usual uniform norm topology. $v$ is a viscosity supersolution of (4) if $v$ is lower semi-continuous in viscosity sense and for all $\left(t_{0}, \omega_{0}\right) \in$ $\mathbb{R}_{+} \times \mathscr{C}\left(\mathbb{R}_{+}, \mathbb{R}^{n}\right)$

- $v$ is bounded from below on $\tilde{D}_{\varepsilon}\left(t_{0}, \omega_{0}\right)$ for some $\varepsilon>0$,

- for all function strictly progressive $\bar{\phi} \in \mathscr{C}_{b}^{1,0,2}\left(\mathbb{R}_{+} \times \Omega \times \mathbb{R}^{n}\right)$ such that $v\left(t_{0}, \omega_{0}\right)=$ $\phi\left(t_{0}, \omega_{0}\right)$, and $\left(t_{0}, \omega_{0}\right)$ is a minimizer of $v-\phi$ on $\tilde{D}_{\varepsilon}\left(t_{0}, \omega_{0}\right)$ for some $\varepsilon>0$,

$$
H\left(u, \omega *_{u} x, \bar{\phi}(u, \omega, x), \partial_{u} \bar{\phi}(u, \omega, x), D_{x} \bar{\phi}(u, \omega, x), D_{x}^{2} \bar{\phi}(u, \omega, x) \geq 0\right.
$$

at point $\left(t_{0}, \omega_{0}, \omega_{0}\left(t_{0}\right)\right)$.

Here $\Omega$ is the set of càdlàg paths with the Skorokhod topology, $\tilde{D}_{\varepsilon}$ is the intersection of $D_{\varepsilon}$ with the set of continuous paths, and $\phi(u, \omega)=\bar{\phi}(u, \omega, \omega(u))$.

The lower semi-continuity property in viscosity sense at $\left(t_{0}, \omega_{0}\right)$ in Definition 4 means

$$
v\left(t_{0}, \omega_{0}\right)=\lim _{\varepsilon \rightarrow 0}\left\{v(s, \omega),(s, \omega) \in \tilde{D}_{\varepsilon}\left(t_{0}, \omega_{0}\right)\right\}
$$


We have a similar definition for a viscosity subsolution. Notice that the continuity along the sets $\tilde{D}_{\varepsilon}$ is also considered in $[13,14]$. However the notion of viscosity solution introduced in $[13,14]$ is fundamentally different from ours.

We will now construct time consistent dynamic risk measures making use of probability measures solution to a path-dependent martingale problem. We will then prove that this leads to viscosity solutions to path-dependent PDEs (2).

\section{Path-dependent Martingale Problem}

In the classical setting, the Feynman Kac formula establishes a link between a solution of a parabolic second order PDE and probability measures solutions to a martingale problem. Assume that $v$ is a solution of the PDE $\partial_{u} v(t, x)+\mathscr{L}^{a}, b v(t, x)=0$, $v(T,)=$.$h with$

$$
\mathscr{L}^{a, b} v(t, x)=\frac{1}{2} \operatorname{Tr}(a(t, x)) D_{x}^{2}(v)(t, x)+b(t, x)^{*} D_{x} v(t, x)
$$

From the Feynman Kac formula, the value $v(t, x)$ can be expressed from the probability measure $Q_{t, x}^{a, b}$ solution to the martingale problem associated to the operator $\mathscr{L}^{a, b}$ starting from $x$ at time $t . v(t, x)=E_{Q_{t, x}^{a, b}}\left(h\left(X_{T}\right)\right)$, where $\left(X_{u}\right)$ is the canonical process.

One natural way to construct soliutions for path-dependent parabolic second order partial differential equations is thus to start with probability measures solution to the path-dependent martingale problem associated to the operator $\mathscr{L}^{a, b}$ for pathdependent coefficients $a$ and $b$. Let $\Omega$ be the set of càdlàg paths and $\left(\mathscr{B}_{t}\right)$ be the canonical filtration. Let $a$ and $b$ be progressively measurable functions on $I R_{+} \times \Omega$ ( $a$ takes values in non negative invertible matrices and $b$ in $I R^{n}$ ). Let $\mathscr{L}^{a, b}$ be the operator defined on $\mathscr{C}_{b}^{2}\left(I R^{n}\right)$ by

$$
\mathscr{L}^{a, b}(t, \omega)=\frac{1}{2} \sum_{1}^{n} a_{i j}(t, \omega) \frac{\partial^{2}}{\partial x_{i} \partial x_{j}}+\sum_{1}^{n} b_{i}(t, \omega) \frac{\partial}{\partial x_{i}}
$$

Definition 5 Let $r \geq 0, \omega_{0} \in \Omega$. A probability measure $Q$ defined on $\left(\Omega,\left(\mathscr{B}_{t}\right)\right)$ is a solution to the path-dependent martingale problem for $\mathscr{L}^{a, b}$ starting from $\omega_{0}$ at time $r$ if

$$
Q\left(\left\{\omega \in \Omega \mid \omega(u)=\omega_{0}(u) \forall 0 \leq u \leq r\right\}\right)=1
$$

and if for all $f \in \mathscr{C}_{b}^{1,2}\left(\mathbb{R}_{+} \times \mathbb{I R}^{n}\right)$, and all $t,\left(Z_{r, t}^{a, b}\right)_{r \leq t}$ given by

$$
Z_{r, t}^{a, b}=f\left(t, X_{t}(\omega)\right)-f\left(r, X_{r}(\omega)\right)-\int_{r}^{t}\left(\frac{\partial}{\partial u}+L^{a, b}(u, \omega)\right)(f)\left(u, X_{u}(\omega)\right) d u
$$

is a $\left(Q,\left(\mathscr{B}_{t}\right)\right)$ martingale. 
In [3] we have studied the more general martingale problem associated with pathdependent diffusions with jumps. We have shown that the good setting to prove that the martingale problem is well posed is to deal with diffusions operators whose coefficients $a$ and $b$ are progressively continuous.

Recall the following result from [3].

Theorem 1 1. Let a be a progressively continuous bounded function defined on $\mathbb{R}_{+} \times \Omega$ with values in the set of non negative matrices. Assume that a $(s, \omega)$ is invertible for all $(s, \omega)$. Let $b$ be a progressively measurable bounded function defined on $\mathbb{R}_{+} \times \Omega$ with values in $\mathbb{R}^{n}$. For all $\left(r, \omega_{0}\right)$, the martingale problem for $\mathscr{L}^{a, a b}$ starting from $\omega_{0}$ at time $r$ is well posed i.e. admits a unique solution $Q_{r, \omega_{0}}^{a, a b}$ on the set of càdlàg paths.

2. Assume furthermore that $b$ is progressively continuous bounded. Consider the set of probability measures $\mathscr{M}_{1}(\Omega)$ equipped with the weak topology. Then the map

$$
(r, \omega, x) \in \mathbb{R}_{+} \times \Omega \times \mathbb{R}^{n} \rightarrow Q_{r, \omega *_{r} x}^{a, a b} \in \mathscr{M}_{1}(\Omega)
$$

is continuous on $\left\{(r, \omega, x) \mid \omega=\omega *_{r} x\right\}$.

\subsection{The Role of Continuous Paths}

In all the following $Q_{r, \omega_{0}}^{a}$ means $Q_{r, \omega_{0}}^{a, 0}$

We start with a result which proves that the probability measure $Q_{r, \omega_{0}}^{a, a b}$ is supported by paths which are continuous after time $r$.

Proposition 1 Every probability measure $Q_{r, \omega_{0}}^{a, a b}$ solution to the martingale problem for $\mathscr{L}^{a, a b}$ starting from $\omega_{0}$ at time $r$ is supported by paths which are continuous after time $r$, i.e. continuous on $[r, \infty[$.

More precisely

$$
Q_{r, \omega_{0}}^{a, a b}\left(\left\{\omega , \omega ( u ) = \omega _ { 0 } ( u ) \forall u \leq r , \text { and } \omega _ { | [ r , \infty [ } \in \mathscr { C } \left(\left[r, \infty\left[, R^{n}\right)\right\}=1\right.\right.\right.
$$

Proof The probability measure $Q_{r, \omega_{0}}^{a, a b}$ is equivalent with $Q_{r, \omega_{0}}^{a}$. Thus we can assume that $b=0$.

The function $a$ is progressively continuous. This means that the function $\bar{a}$ is continuous. Let $a_{n}$ be the $\frac{1}{n}$ delayed function defined as $\bar{a}_{n}(u, \omega, x)=\bar{a}\left(u-\frac{1}{n}, \omega, x\right)$ for all $u \geq r+\frac{1}{n}$ and $\bar{a}_{n}(u, \omega, x)=\bar{a}(r, \omega, x)$ for all $0 \leq u \leq r+\frac{1}{n}$. The function $a_{n}$ is also progressively continuous. Given $n$, let $t_{k}^{n}$ be an increasing sequence such that $t_{0}^{n}=r$ and $\left|t_{k+2}^{n}-t_{k}^{n}\right|<\frac{1}{n}$.

On a Polish space for every subsigma algebra of the Borel sigma algebra there exists a regular conditional probability distribution. It follows from [22] and the uniqueness of the solution for $\mathscr{L}^{a_{n}, 0}$ starting from $\omega$ at time $t_{k}^{n}$, that for all $t_{k}^{n}$, $Q_{t_{k}^{n}, \omega}^{a_{n}}(\xi)=E_{Q_{r, \omega_{0}}^{a_{n}}}\left(\xi \mid \mathscr{B}_{t_{k}^{n}}\right)(\omega)$ for $Q_{r, \omega_{0}}^{a_{n}}$ almost all $\omega$. Let $a_{n, \omega}(u, x)=\bar{a}_{n}(u, \omega, x)$ 
Let $A_{k}=\left\{\omega^{\prime}, \omega_{\left[t_{k}^{n}, t_{k+2}^{n}[\right.}^{\prime} \in \mathscr{C}\left(\left[t_{k}^{n}, t_{k+2}^{n}[)\right\}\right.\right.$. Given $\omega$ the function $a_{n, \omega}$ is not pathdependent. It follows then from [11] that $Q_{t_{k}^{n}, \omega}^{a_{n, \omega}}$ is supported by paths continuous on $\left[t_{k}^{n}, \infty\left[\right.\right.$. We remark that $\bar{a}_{n}\left(u, \omega^{\prime}, x\right)=a_{n, \omega}(u, x)$ for all $t_{k}^{n} \leq u \leq t_{k+2}^{n}$ and all $\omega^{\prime}$ such that $\omega^{\prime}(u)=\omega(u)$ for all $u \leq t_{k}^{n}$. It follows that $Q_{t_{k}^{n}, \omega}^{a_{n}}\left(A_{k}\right)=1$ for all $\omega$. We deduce by induction that $Q_{r, \omega_{0}}^{a_{n}}\left(\left\{\omega^{\prime}, \omega_{\mid[r, \infty[}^{\prime} \in \mathscr{C}\left(\left[r, \infty\left[, \mathbb{R}^{n}\right)\right\}=1\right.\right.\right.$.

The $a_{n}$ being uniformly bounded, for given $r$ and $\omega_{0}$, the set of probabilty measures $\left\{Q_{r, \omega_{0}}^{a_{n}}, n \in N^{*}\right\}$ is weakly relatively compact. There is a subsequence weakly converging to a probability measure $Q$. From the continuity assumption on $a$, it follows that $Q$ solves the martingale problem for $\mathscr{L}^{a, 0}$ starting from $\omega_{0}$ at time $r$. The uniqueness of the solution to this martingale problem implies that $Q=Q_{r, \omega_{0}}^{a}$. The set $\left\{\omega^{\prime}, \quad \omega_{\mid[r, \infty[}^{\prime} \in \mathscr{C}\left(\left[r, \infty\left[, \mathbb{R}^{n}\right)\right\}\right.\right.$ is a closed subset of $\Omega$. It follows from the Portmanteau Theorem, see e.g. [2] Theorem 2.1, that $Q_{r, \omega_{0}}^{a}\left(\left\{\omega^{\prime}, \omega_{\mid[r, \infty[}^{\prime} \in\right.\right.$ $\mathscr{C}\left(\left[r, \infty\left[, I^{n}\right)\right\}=1\right.$

Corollary 1 For all continuous path $\omega_{0}$ and all $r$, the support of the probability measure $Q_{r, \omega_{0}}^{a, a b}$ is contained in the set of continuous paths:

$$
Q_{r, \omega_{0}}^{a, b} \mathscr{C}\left(\left[I R_{+}, \mathbb{R}^{n}\right)\right)=1
$$

Remark 1 In the simpler case where the function $a$ is only defined on the set of continuous paths, the continuity hypothesis is just the usual continuity hypothesis for a function defined on $\mathbb{R} R_{+} \times \mathscr{C}\left(\left[\mathbb{R}_{+}, \mathbb{I}^{n}\right)\right)$ for the uniform norm topology. The associated martingale problem: probability measure solution to the martingale problem for $\mathscr{L}^{a, 0}$ starting from $\omega_{0}$ at time $r$ can only be stated for initial continuous paths $\omega_{0}$ (otherwise the path-dependent function $a(u, \omega)$ should be defined for paths $\omega$ which can have jumps before time $u$ ).

\section{Stable Set of Probability Measures Solution to a Path-dependent Martingale Problem}

In all the paper $\Omega$ denotes the set of càdlàg paths endowed with the Skorokhod topology. From now on, $a(s, \omega)$ is a given progressively continuous function on $R_{+} \times \Omega$ with values in non negative matrices. We assume that $a$ is bounded and that $a(s, \omega)$ is invertible for all $(s, \omega)$. The explicit construction of dynamic risk measures developed here, making use of probability measures solutions to a martingale problem was first initiated in the unpublished preprint [4] in the Markovian case. We have introduced in [5] a general method to construct time consistent convex dynamic risk measures. This construction makes use of two tools. The first one is a set $\mathscr{Q}$ of equivalent probability measures stable by composition and stable by bifurcation (cf. [5] Definition 4.1). The second one consists in penalties $\alpha_{s, t}(Q), s \leq t$ defined for every probability measure $Q$ in $\mathscr{Q}$, satisfying the local condition and the cocycle condition. The corresponding definitions are recalled in the Appendix. 


\subsection{Multivalued Mapping and Continuous Selector}

Definition $6 X$ denotes the quotient of $I R_{+} \times \Omega \times I R^{n}$ by the equivalence relation $\sim:(t, \omega, x) \sim\left(t^{\prime}, \omega^{\prime}, x^{\prime}\right)$ if $t=t^{\prime}, x=x^{\prime}$ and $\omega(u)=\omega^{\prime}(u) \forall u<t$. The metric topology on $X$ is induced by the one to one map from $X$ into a subset of $\mathbb{R}_{+} \times \Omega \times \mathbb{R}^{n}$ : $(t, \omega, x) \rightarrow\left(t, \omega *_{t} x, x\right)$, where $\omega *_{t} x$ has been defined in Sect. 2.2 Eq. (3).

The following observation is straightforward.

Remark 2 The set $X$ is equipped with the metric topology defined above. Then every progressively continuous map on $\mathbb{R}_{+} \times \Omega$ defines a unique continuous map on $X$. Furthermore every map continuous on the subset $\left\{(r, \omega, x), \omega=\omega *_{r} x\right\}$ of $\mathbb{R}_{+} \times$ $\Omega \times \mathbb{R}^{n}$ defines also a unique continuous map on $X$.

Recall now the definition of a multivalued mapping from $X$ to $Y$. We use here the terminology chosen in [9]. Notice that the terminology used in [1] for multivalued mapping is correspondence.

Definition 7 A multivalued mapping $\Lambda$ from $X$ into $I^{n}$ is a map $\Lambda$ defined on $X$ such that for all $(t, \omega, x) \in X, \Lambda(t, \omega, x)$ is a subset of $\mathbb{I R}^{n}$. It can have additional properties:

1. $\Lambda$ is convex if $\forall(t, \omega, x) \in X, \Lambda(t, \omega, x)$ is a convex subset of $\mathbb{I R}^{n}$.

2. $\Lambda$ is closed if for all $(t, \omega, x), \Lambda(t, \omega, x)$ is closed.

Recall the following definition of a continuous selector (Definition 16.57 of [1]).

Definition 8 A selector from a multivalued mapping $\Lambda$ from $\mathrm{X}$ into $\mathbb{I}^{n}$ is a function $s: X \rightarrow \mathbb{R}^{n}$ such that $s(t, \omega, x) \in \Lambda(t, \omega, x)$ for all $(t, \omega, x) \in X$. A continuous selector is a selector which is continuous.

Recall the following definition from [1] (Definition 16.2 and Lemma 16.5):

Definition 9 A multivalued mapping $\Lambda$ from $\mathrm{X}$ into $\mathbb{I}^{n}$ is lower hemicontinuous if it satisfies the following equivalent conditions

- For every closed subset $\mathrm{F}$ of $\mathbb{I R}^{n}, \Lambda^{u}(F)=\{(t, \omega, x) \in X: \Lambda(t, \omega, x) \subset F\}$ is closed

- For every open subset $\mathrm{V}$ of $\mathbb{I R}^{n}, \Lambda^{l}(V)=\{(t, \omega, x) \in X: \Lambda(t, \omega, x) \cap V \neq \emptyset\}$ is open

Recall the following Michael Selection Theorem (cf. [1] Theorem 16.61)

Theorem 2 A lower hemicontinuous mapping from a paracompact space into a Banach space with non empty closed convex values admits a continuous selector.

Recall also that every metrizable space is paracompact (Theorem 2.86 of [1]). 


\subsection{Stable Set of Probability Measures Associated to a Multivalued Mapping}

In all the following, $\Lambda$ is a closed convex lower hemicontinuous multivalued mapping from $X$ into $I^{n}$. In the following $\left(X_{t}\right)$ denotes the canonical process on $\Omega$ : For all càdlàg path $\omega, X_{t}(\omega)=\omega(t)$. $\left(\mathscr{B}_{t}\right)$ is the canonical filtration.

Given the progressively continuous matrix valued map $a$, given $r \geq 0$, and $\omega \in \Omega$, we want to associate to $\Lambda$ a stable set $\mathscr{Q}_{r, \omega}(\Lambda)$ of probability measures on $(\Omega, \mathscr{B})$ all equivalent with the probability measure $Q_{r, \omega}^{a}$ on $\mathscr{B}_{t}$. Furthermore we want to construct a continuous function $v$ on $X$. Therefore we start with continuous selectors $\bar{\lambda}$ from $\Lambda$.

Definition 10 Let $a$ be progressively continuous bounded defined on $I R_{+} \times \Omega$ with values in non negative matrices, such that $a(t, \omega)$ is invertible for all $(t, \omega)$. Let $\Lambda$ be a closed convex lower hemicontinuous multivalued mapping from $X$ into $\mathbb{R}^{n}$.

- We define $L(\Lambda)$ to be the set of continuous bounded selectors from the multivalued mapping $\Lambda$.

- For given $r \geq 0$ and $\omega \in \Omega$, the set $\tilde{\mathscr{Q}}_{r, \omega}(\Lambda)$ is the stable set of probability measures generated by the probability measures $Q_{r, \omega}^{a, a \lambda}, \bar{\lambda} \in L(\Lambda)$ with $\lambda\left(t, \omega^{\prime}\right)=$ $\bar{\lambda}\left(t, \omega^{\prime}, X_{t}\left(\omega^{\prime}\right)\right)$

$$
\left(\frac{d Q_{r, \omega}^{a, a \lambda}}{d Q_{r, \omega}^{a}}\right)_{\mathscr{B}_{T}}=\exp \left[\int_{r}^{T}\left\langle\lambda\left(t, \omega^{\prime}\right), d X_{t}\right\rangle-\frac{1}{2} \int_{r}^{T}\left\langle\lambda\left(t, \omega^{\prime}\right), a\left(t, \omega^{\prime}\right) \lambda\left(t, \omega^{\prime}\right)\right\rangle d t\right]
$$

We give now a description of the set $\tilde{\mathscr{Q}}_{r, \omega}(\Lambda)$.

Definition 11 We define $\tilde{L}(\Lambda)$ to be the set of processes $\bar{\mu}$ such that there is a finite subdivision $0=s_{0}<\cdot<s_{i}<s_{i+1} \cdots<s_{k}<\infty$. There is a continuous selector $\bar{\lambda}_{0, i_{0}}$ in $L(\Lambda)$. And for all $0<i \leq k$ there is a finite partition $\left(A_{i, j}\right)_{j \in I_{i}}$ of $\Omega$ into $\mathscr{B}_{s_{i}}$ measurable sets, and continuous selectors $\bar{\lambda}_{i, j}$ in $L(\Lambda)$ such that

$$
\begin{array}{r}
\forall s_{i}<u \leq s_{i+1}, \forall \omega^{\prime} \in \Omega, \bar{\mu}\left(u, \omega^{\prime}, x\right)=\sum_{j \in I_{i}} \bar{\lambda}_{i, j}\left(u, \omega^{\prime}, x\right) 1_{A_{i, j}}\left(\omega^{\prime}\right) \\
\forall s_{k}<u \forall \omega^{\prime} \in \Omega, \bar{\mu}\left(u, \omega^{\prime}, x\right)=\sum_{j \in I_{k}} \bar{\lambda}_{k, j}\left(u, \omega^{\prime}, x\right) 1_{A_{k, j}}\left(\omega^{\prime}\right) \\
\forall u \leq s_{0}, \forall \omega^{\prime} \in \Omega, \bar{\mu}\left(u, \omega^{\prime}, x\right)=\bar{\lambda}_{0, i_{0}}\left(u, \omega^{\prime}, x\right)
\end{array}
$$

Remark 3 Every process $\bar{\mu}$ in $\tilde{L}(\Lambda)$ is bounded strictly progressive and $\mathscr{P} \times \mathscr{B}\left(\mathbb{R}^{n}\right)$ measurable where $\mathscr{P}$ is the predictable sigma algebra. However there is no uniform bound. 
Proposition 2 1. Let a be as above and $\bar{\mu} \in \tilde{L}(\Lambda)$. For all $r \geq 0$ and all $\omega \in \Omega$, there is a unique solution to the martingale problem for $\bar{L}^{a, a \mu}$ starting from $\omega$ at time $r$ with $\mu(u, \omega)=\bar{\mu}(u, \omega, \omega(u))$. Furthermore for all $r<s$, the map $\omega^{\prime} \rightarrow Q_{s, \omega^{\prime}}^{a, a \mu}$ is $\mathscr{B}_{s}$ measurable and is a regular conditional probability distribution of $Q_{r, \omega}^{a, a \mu}$ given $\mathscr{B}_{s}$.

2. Given $0 \leq r$, the set $\tilde{\mathscr{Q}}_{r, \omega}(\Lambda)$ is the set of all probability measures $Q_{r, \omega}^{a, a \mu}$ for some process $\bar{\mu}$ belonging to $\tilde{L}(\Lambda)$.

Proof Let $\bar{\mu} \in \tilde{L}(\Lambda)$. There is a finite subdivision $0=s_{0}<\cdots<s_{i}<s_{i+1}<$ $\cdots s_{k}<\infty$ such that $\bar{\mu}$ is described by Eq. (13). Let $r$ and $\omega$. We prove first by induction on $k$ that there is a unique solution $Q$ to the martingale problem for $\mathscr{L}^{a, a \mu}$ starting from $\omega$ at time $r$ and that $Q$ belongs to $\tilde{\mathscr{Q}}_{r, \omega}(\Lambda)$.

For $k=0$ the result is true by hypothesis.

Inductive step: Assume that $k \geq 1$ and that the result is proved for $k-1$. Let $Q$ be a solution to the martingale problem for $\mathscr{L}^{a, a \mu}$ starting from $\omega$ at time $r$. Let $Q_{s_{k}, \omega^{\prime}}$ be a regular conditional probability distribution of $Q$ given $\mathscr{B}_{s_{k}}$. From [22] it follows that for $Q$ almost all $\omega^{\prime}$ in $A_{k, j}, Q_{s_{k}, \omega^{\prime}}$ is a solution to the martingale problem for $\mathscr{L}^{a, a \lambda_{k, j}}$ starting from $\omega^{\prime}$ at time $s_{k}$. The martingale problem for $\mathscr{L}^{a, a \lambda_{k, j}}$ is well posed. Let $Q_{s_{k}, \omega^{\prime}}^{a, a \lambda_{k j}}$ be the unique solution to the martingale problem for $\mathscr{L}^{a, a \lambda_{k, j}}$ starting from $\omega^{\prime}$ at time $s_{k}$. It follows that $Q_{s_{k}, \omega^{\prime}}=Q_{s_{k}, \omega^{\prime}}^{a, a \lambda_{k, j}}$ on $A_{k, j} Q$ a.s. Thus for all $\xi$,

$$
\begin{aligned}
E_{Q}\left(\xi \mid \mathscr{B}_{s_{k}}\right)\left(\omega^{\prime}\right) & =\sum_{j \in I_{k}} 1_{A_{k, j}}\left(\omega^{\prime}\right) Q_{s_{k}, \omega^{\prime}}^{a, a \lambda_{k, j}}(\xi) \\
E_{Q}\left(\xi \mid \mathscr{B}_{s_{k}}\right) & =\sum_{j \in I_{k}} 1_{A_{k, j}} E_{Q_{r, \omega^{\prime}}^{a, a \lambda_{k, j}}}\left(\xi \mid \mathscr{B}_{s_{k}}\right)
\end{aligned}
$$

On the other hand the restriction of $Q$ to $\mathscr{B}_{s_{k}}$ is a solution to the martingale problem for $\mathscr{L}^{a, a v}$ where $v \in \tilde{L}(\Lambda)$ is associated to the subdivision $\left(s_{i}\right)_{0 \leq i \leq k-1}$ and $v$ coincides with $\mu$ on $\mathscr{B}_{s_{k}}$. From the induction hypothesis it follows that the restriction of $Q$ to $\mathscr{B}_{s_{k}}$ is uniquely determined, it coincides with $Q_{r, \omega}^{a, a v}$ and it belongs to $\tilde{\mathscr{Q}}_{r, \omega}(\Lambda)$.

The end of the proof of the inductive step follows then from Eq. (14), from the $\mathscr{B}_{s_{k}}$ measurability of the map $\omega^{\prime} \rightarrow Q_{s_{k}, \omega^{\prime}}^{a, a \lambda_{k, j}}(\xi)$ and from the definition of $\tilde{\mathscr{Q}}_{r, \omega}(\Lambda)$.

On the other hand it is easy to verify that the set $\left\{Q_{r, \omega}^{a, a \mu}: \bar{\mu} \in \tilde{L}(\Lambda)\right\}$ is stable.

\section{Construction of Penalties}

In the preceding section we have constructed for all given $(r, \omega)$ a stable set of probability measures $\tilde{\mathscr{Q}}_{r, \omega}(\Lambda)$ associated to a multivalued mapping $\Lambda$. In this section we construct penalties $\alpha_{s t}(Q)$ for all $r \leq s \leq t$ and all $Q \in \tilde{\mathscr{Q}}_{r, \omega}(\Lambda)$ making use of a function $g$. 
Let $g: \mathbb{R}^{+} \times \Omega \times \mathbb{R}^{n} \rightarrow \mathbb{R} \cup\{+\infty\}$ be a progressively measurable function. Let $\Lambda$ be a closed convex multivalued Borel mapping such that for all $(t, \omega, x) \in \mathbb{R}^{+} \times \Omega \times \mathbb{R}^{n}$, $\{0\} \subset \Lambda(t, \omega, x) \subset\left\{y \in \mathbb{R}^{n} \mid g\left(t, \omega *_{t} x, y\right)<\infty\right\}$. Define $f$ as follows:

$$
\forall z \in \mathbb{R}^{d} f(t, \omega, z)=\sup _{y \in \Lambda\left(t, \omega, X_{t}(\omega)\right)}(-z . y-g(t, \omega, y))
$$

The following lemma is straightforward:

Lemma 1 For all $(t, \omega), f(t, \omega,$.$) is a closed convex function which is the dual$ transform of the function $\tilde{g}(t, \omega,$.$) where$

$$
\begin{aligned}
\tilde{g}(t, \omega, y) & =g(t, \omega, y) \text { if } y \in \Lambda\left(t, \omega, X_{t}(\omega)\right) \\
& =+\infty \text { else }
\end{aligned}
$$

For every $(t, \omega) \operatorname{dom}(\tilde{g}(t, \omega,))=.\Lambda\left(t, \omega, X_{t}(\omega)\right)$

If $g(t, \omega, 0)=0 \forall(t, \omega)$, $f$ takes values in $[0, \infty]$.

If $g$ takes values in $[0, \infty]$ and satisfies $\forall(t, \omega)$, inf $f_{y \in \Lambda\left(t, \omega, X_{t}(\omega)\right)} g(t, \omega, y)=0$ then for all $(t, \omega), f(t, \omega, 0)=0$.

Notice that, since $\Lambda$ is a closed convex multivalued mapping, replacing $g$ by $\tilde{g}$, one can always assume that for all $(t, \omega), \operatorname{dom}\left(g(t, \omega,)=.\left\{y \in \mathbb{R}^{d} \mid g(t, \omega, y)<\infty\right\}\right.$ is closed, convex and equal to $\Lambda\left(t, \omega, X_{t}(\omega)\right)$. We assume this in all the remainder.

Definition 12 1. $g$ satisfies the following polynomial growth condition (GC1) if there is $K>0, m \in N^{*}$ and $\varepsilon>0$ such that

$$
\forall y \in \Lambda\left(u, \omega, X_{u}(\omega)\right),|g(u, \omega, y)| \leq K\left(1+\sup _{s \leq u}\left\|X_{s}(\omega)\right\|\right)^{m}\left(1+\|y\|^{2-\varepsilon}\right)
$$

2. $g$ satisfies the growth condition (GC2) if there is $K>0$ such that

$$
\forall y \in \Lambda\left(u, \omega, X_{u}(\omega)\right),|g(u, \omega, y)| \leq K\left(1+\|y\|^{2}\right)
$$

Recall the following definition of BMO processes.

Definition 13 Let $C>0$. Let $P$ be a probability measure. A progressively measurable process $\mu$ belongs to $B M O(P)$ and has a BMO norm less or equal to $C$ if for all stopping times $\tau$,

$$
E_{P}\left(\int_{\tau}^{\infty}|| \mu_{s} \|^{2} d s \mid \mathscr{F}_{\tau}\right) \leq C
$$

Recall also from [15] that the stochastic exponential $\mathscr{E}(\mu)$ of a BMO process $\mu$ is uniformly integrable, and that the BMO norms with respect to $P$ and $P(\mathscr{E}(\mu)$.) are equivalent. Also from [15], for all $C>0$ there is $1<p_{0}<\infty$ such that for all 
$B M O(P)$ process $\mu$ with $\|\mu\|_{B M O(P)} \leq C$, the stochastic exponential $\mathscr{E}(\mu)$ satisfies the reverse Hölder inequality:

$$
\left[E_{P}\left(\mathscr{E}(\mu)^{p_{0}} \mid \mathscr{B}_{S}\right)\right]^{\frac{1}{p_{0}}} \leq K_{C} \mathscr{E}(\mu)_{s}
$$

Definition 14 Assume that $g$ is non negative or satisfies one of the growth conditions (GC1) or (GC2). Let $0 \leq r \leq T$. For all $B M O\left(Q_{r, \omega}^{a}\right)$ process $\mu \Lambda$-valued, for all $r \leq s \leq t \leq T$, define the penalty $\alpha_{s, t}\left(Q_{r, \omega}^{a, a \mu}\right)$ as follows

$$
\alpha_{s, t}\left(Q_{r, \omega}^{a, a \mu}\right)=E_{Q_{r, \omega}^{a, a \mu}}\left(\int_{s}^{t} g(u, \omega, \mu(u, \omega)) d u \mid \mathscr{B}_{s}\right)
$$

We need to verify that the penalties are well defined for all BMO processes and that they satisfy the local property and the cocycle condition. (Definition introduced in [5], Definition 4.3 and recalled in the Appendix).

Proposition 3 Assume that the process $\mu$ belongs to $B M O\left(Q_{r, \omega}^{a}\right)$. Let $C$ such that $\|\mu\|_{B M O\left(Q_{r, \omega}^{a}\right)} \leq C$.

-1. Assume that $g$ satisfies the growth condition (GC1). Then Eq.(20) defines a random variable in $L_{p}\left(Q_{r, \omega}^{a}\right)$ for all $1 \leq p<\infty$, and for given $p$, the $L_{p}\left(Q_{r, \omega}^{a}\right)$ norms of $\alpha_{s, t}\left(Q_{r, \omega}^{a, a \mu}\right)$ are uniformly bounded for $r \leq s \leq t \leq T$, for all $\mu$ such that $\|\mu\|_{B M O\left(Q_{r, \omega}^{a}\right)} \leq C$.

$\alpha_{s, t}\left(Q_{r, \omega}^{a, a \mu}\right)$ belongs also to $L_{1}\left(Q_{r, \omega}^{a, a \mu}\right)$ and the $L_{1}\left(Q_{r, \omega}^{a, a \mu}\right)$ norms of $\alpha_{s, t}\left(Q_{r, \omega}^{a, a \mu}\right)$ are uniformly bounded for $\|\mu\|_{B M O\left(Q_{r, \omega}^{a}\right)} \leq C$ and $r \leq s \leq t \leq T$.

2. Assume that $g$ satisfies (GC2), then the random variables $\alpha_{s, t}\left(Q_{r, \omega}^{a, a \mu}\right)$ belong to $L_{\infty}\left(Q_{r, \omega}^{a}\right)$ and are uniformly bounded for $\|\mu\|_{B M O\left(Q_{r, \omega}^{a}\right)} \leq C$ and $r \leq s \leq t \leq$ $T$.

3. In case $g$ is non negative, Eq.(20) defines a non negative $\mathscr{B}_{s}^{r}$ random variable.

- Assume that $g$ satisfies the growth condition (GC1) or (GC2). Then the penalty defined in (20) satisfies the cocycle condition for every $Q_{r, \omega}^{a, a \mu}$ : Let $r \leq s \leq t \leq u$

$$
\alpha_{s, u}\left(Q_{r, \omega}^{a, a \mu}\right)=\alpha_{s, t}\left(Q_{r, \omega}^{a, a \mu}\right)+E_{Q_{r, \omega}^{a, a \mu}}\left(\alpha_{t, u}\left(Q_{r, \omega}^{a, a \mu}\right) \mid \mathscr{B}_{s}\right)
$$

- The penalty defined in (20) is local on $\tilde{\mathscr{Q}}_{r, \omega}(\Lambda)$

- If $g(t, \omega, 0)=0 \forall(t, \omega) \in \mathbb{R}^{+} \times \Omega$, The probability measure $Q_{r, \omega}^{a}$ has zero penalty.

Proof 1. Assume that the function $g$ satisfies the growth condition (GC1). Without loss of generality one can assume that $m \geq 2$. Choose $p_{1}>1$ such that $(2-$ $\varepsilon) p_{1}=2$. Let $q$ be the conjugate exponent of $p_{1}$. 
It follows from the conditional Hölder inequality and the equivalence of the BMO norms with respect to $Q_{r, \omega}^{a}$ and $Q_{r, \omega}^{a, a \mu}$ that

$$
\begin{aligned}
& E_{Q_{r, \omega}^{a, a \mu}}\left(\int_{s}^{t}\left(1+\sup _{s \leq u}\left\|X_{s}(\omega)\right\|\right)^{m}\left\|\mu_{u}(\omega)\right\|^{2-\varepsilon} d u \mid \mathscr{B}_{s}\right) \\
\leq & K_{1} C^{\frac{1}{p_{1}}}\left[E_{Q_{r, \omega}^{a, a \mu}}\left(\sup _{s \leq s^{\prime} \leq u}\left(1+\| X_{s^{\prime}}||\right)^{m q}\right) \mid \mathscr{B}_{s}\right]^{\frac{1}{q}}(t-s)^{\frac{1}{q}}
\end{aligned}
$$

Let $p_{0}$ be such that Eq. (19) is satisfied for $P=Q_{r, \omega}^{a}$. Let $q_{0}$ be the conjugate exponent of $p_{0}$. It follows from conditional Hölder inequality and (19) that

$$
\begin{gathered}
\left.E_{Q_{r, \omega}^{a, a \mu}}\left(\sup _{s^{\prime} \leq u}\left(1+\| X_{s^{\prime}}||\right)^{m q} \mid \mathscr{B}_{s}\right)\right) \leq K_{C} E_{Q_{r, \omega}^{a}}\left(\sup _{s \leq s^{\prime} \leq t}\left(1+|| X_{s^{\prime}}||\right)^{m q q_{0}} \mid \mathscr{B}_{s}\right)^{\frac{1}{q_{0}}} \\
\leq K_{C} E_{Q_{r, \omega}^{a}}\left(\sup _{s \leq s^{\prime} \leq t}\left(1+\| X_{s^{\prime}}||\right)^{m q j q_{0}} \mid \mathscr{B}_{s}\right)^{\frac{1}{q_{0} j}}
\end{gathered}
$$

for all $j \geq 1$. The first assertion of 1 of the proposition follows then from the Eqs. (22) and (23) and the inequality $\left.E_{Q_{r, \omega}^{a}}\left(\sup _{s \leq u \leq t}\left(1+\left\|X_{t}\right\|\right)^{k}\right)\right)<\infty$ for all $k \geq 2$ ( [16], Chap. 2 Sect. 5).

The second asssertion of 1. of the proposition follows from Eq. (22) and then from Eq. (23) applied with $\mathscr{B}_{s}$ equal to the trivial sigma algebra.

2. Assume that $g$ satisfies the the growth condition (GC2). Thus

$$
\left|\alpha_{s t}\left(Q_{r, \omega}^{a, a \mu}\right)\right| \leq K E_{Q_{r, \omega}^{a, a \mu}}\left(\int_{s}^{t}\left(1+|| \mu_{u}(\omega) \|^{2}\right) d u \mid \mathscr{B}_{s}\right)
$$

The result follows then from the BMO condition.

3. The case $g$ non negative is trivial.

- The cocycle condition (21) follows easily from the definition (20) and the above integrability.

- We prove now that the penalty $\alpha$ is local on $\tilde{\mathscr{Q}}_{r, \omega}(\Lambda)$. Let $\bar{\mu}, \bar{v} \in \tilde{L}(\Lambda)$. The probability measures $Q_{r, \omega}^{a, a \mu}$ and $Q_{r, \omega}^{a, a v}$ are equivalent to $Q_{r, \omega}^{a}$. Let $r \leq s \leq t$ and $A$ be $\mathscr{B}_{s}$-measurable. Assume that for all $X$ in $L^{\infty}\left(\mathscr{B}_{t}\right), E_{Q_{r, \omega}^{a, a \mu}}\left(X \mid \mathscr{B}_{s}\right) 1_{A}=$ $E_{Q_{r, \omega}^{a, a v}}\left(X \mid \mathscr{B}_{S}\right) 1_{A}$. It follows from the equality $\frac{\mathscr{E}(a \mu)_{t}}{\mathscr{E}(a \mu)_{s}} 1_{A}=\frac{\mathscr{E}(a v)_{t}}{\mathscr{E}(a v)_{s}} 1_{A}$ and the $\mathscr{P} \times$ $\mathscr{B}\left(\mathbb{R}^{n}\right)$ measurability of $\bar{\mu}$ and $\bar{v}$ that $1_{\mid] s, t[} 1_{A} \mu=1_{\mid] s, t[} 1_{A} v Q_{r, \omega}^{a}$ a.s. From (20) we get $\alpha_{s, t}\left(Q_{r, \omega}^{a, a \mu}\right) 1_{A}=\alpha_{s, t}\left(Q_{r, \omega}^{a, a v}\right) 1_{A}$. Thus the penalty $\alpha$ is local on $\tilde{\mathscr{Q}}_{r, \omega}(\Lambda)$.

- The last point follows easily from the definition of the penalty. 


\section{Time Consistent Dynamic Risk Measures Associated to Path-dependent Martingale Problems}

We change the sign in the classical definition of risk measures in order to avoid the minus sign which appears in the time consistency property for usual dynamic risk measures. In fact $\rho_{s t}(-X)$ are "usual" dynamic risk measures.

\subsection{Normalized Time-Consistent Convex Dynamic Risk Measures}

Proposition 4 Let $\tilde{\mathscr{Q}}_{r, \omega}(\Lambda)$ be the stable set of probability measures defined in Definition 10. Assume that $g$ is non negative, and that for all $\left(u, \omega^{\prime}\right), g\left(u, \omega^{\prime}, 0\right)=0$. Let $r \leq s \leq t$. The formula

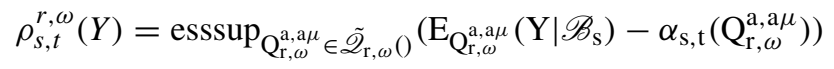

where $\alpha_{s, t}\left(Q_{r, \omega}^{a, a \mu}\right)$ is given by Eq. (20) defines a normalized time consistent convex dynamic risk measure on $L^{\infty}\left(\Omega, \mathscr{B}, Q_{r, \omega}^{a}\right)$.

For given $0 \leq r \leq t$ and $Y$ in $L^{\infty}\left(\Omega^{r}, \mathscr{B}_{t}^{r}, Q_{r, y}^{a}\right)$, the process $\left(\rho_{s, t}^{r, \omega}(Y)\right)_{r \leq s \leq t}$ admits a càdlàg version.

Proof Notice that for all bounded $Y$,

$$
-\|Y\|_{\infty} \leq E_{Q_{r, \omega}^{a}}\left(Y \mid \mathscr{B}_{S}\right) \leq \rho_{s, t}^{r, \omega}(Y) \leq \operatorname{esssup}_{\mathrm{Q}_{\mathrm{r}, \omega}^{\mathrm{a}, a} \in \tilde{\mathscr{Q}}_{\mathrm{r}, \omega}()}\left(\mathrm{E}_{\mathrm{Q}_{\mathrm{r}, \omega}^{\mathrm{a}, a} \mu}\left(\mathrm{Y} \mid \mathscr{B}_{\mathrm{S}}\right) \leq\|\mathrm{Y}\|_{\infty}\right.
$$

Thus for all $r \leq s \leq t,\left\|\rho_{s, t}^{r, \omega}(Y)\right\|_{\infty} \leq\|Y\|_{\infty}$. The first statement follows then from Definition 10, from Propositions 2 and 3 and from Theorem 4.4 of [5].

The proof of the regularity of paths which was given in [6] Theorem 3 for normalized convex dynamic risk measures time consistent for stopping times can be extended to normalized convex dynamic risk measures time consistent for deterministic times.

We have the following extension of the dynamic risk measure to random variables essentially bounded from below:

Corollary 2 The definition of $\rho_{s, t}^{r, \omega}(Y)$ can be extended to random variables $Y\left(\mathscr{B}_{t}\right)$ measurable which are only essentially bounded from below.

$\rho_{s, t}^{r, \omega}(Y)=\lim _{n \rightarrow \infty} \rho_{s, t}^{r, \omega}(Y \wedge n)$. For every $Y$ essentially bounded from below, the process $\left(\rho_{s, t}^{r, \omega}(Y)\right)$ is optional.

Proof Let $Y$ be $\mathscr{B}_{t}$-measurable and $Q_{r, \omega}^{a}$-essentially bounded from below, $Y$ is the increasing limit of $Y_{n}=Y \wedge n$ as $n$ tends to $\infty$. Define $\rho_{s, t}^{r, \omega}(Y)$ as the increasing limit of $\rho_{s, t}^{r, \omega}\left(Y_{n}\right)$. As we already know that for given $s$ and $t, \rho_{s, t}^{r, \omega}(Y)$ defined on bounded 
random variables by formula (24) is continuous from below, the extended definition coincides with the previous one on $Q_{r, \omega}^{a}$-essentially bounded random variables. From Proposition 4 for every $n$ one can choose a càdlàg version of the process $\rho_{s, t}^{r, \omega}\left(Y_{n}\right)$. Thus the map $(s, \omega) \rightarrow \rho_{s, t}^{r, \omega}(Y)=\lim \rho_{s, t}^{r, \omega}\left(Y_{n}\right)$ is measurable for the optional sigma algebra.

\subsection{General Time-Consistent Convex Dynamic Risk Measures}

In this section the function $g$ (and thus the penalty) is not assumed to be non negative.

Definition 15 Let $Q$ be a probability measure on $\left(\Omega,\left(\mathscr{B}_{t}\right)_{t \in \mathbb{R}_{+}}\right)$. The multivalued mapping $\Lambda$ is $B M O(Q)$ if there is a map $\phi \in B M O(Q)$ such that

$$
\forall(u, \omega), \sup \{\|y\|, \quad y \in \Lambda(u, \omega)\} \leq \phi(u, \omega)
$$

In the following, $p_{0}$ is chosen such that the reverse Hölder inequality (19) is satisfied for $a \phi$.

Theorem 3 Let $(r, \omega)$. Assume that the multivalued set $\Lambda$ is $B M O\left(Q_{r, \omega}^{a}\right)$. Let $\tilde{\mathscr{Q}}_{r, \omega}(\Lambda)$ be the stable set of probability measures defined in Definition 10.

Let $r \leq s \leq t$. Let

$$
\rho_{s, t}^{r, \omega}(Y)=\operatorname{essup}_{\mathrm{Q}_{\mathrm{r}, \omega}^{\mathrm{a}, \mu} \in \tilde{\mathscr{Q}}_{\mathrm{r}, \omega}()}\left(\mathrm{E}_{\mathrm{Q}_{\mathrm{r}, \omega}^{\mathrm{a}, a} \mu}\left(\mathrm{Y} \mid \mathscr{B}_{\mathrm{S}}\right)-\alpha_{\mathrm{s}, \mathrm{t}}\left(\mathrm{Q}_{\mathrm{r}, \omega}^{\mathrm{a}, \mathrm{a} \mu}\right)\right)
$$

where $\alpha_{s, t}\left(Q_{r, \omega}^{a, a \mu}\right)$ is given by Eq. (20)

- Assume that $g$ satisfies the growth condition (GC1). The above Eq.(25) defines a dynamic risk measure $\left(\rho_{s, t}^{r, \omega}\right)$ on $L_{p}\left(Q_{r, \omega}^{a},\left(\mathscr{B}_{t}\right)\right)$ for all $q_{0} \leq p<\infty$, (where $q_{0}$ is the conjugate exponent of $p_{0}$ chosen as above). These dynamic risk measures are time consistent for stopping times taking a finite number of values.

- Assume that $g$ satisfies the growth condition (GC2). The above Eq.(25) defines a dynamic risk measure $\left(\rho_{s, t}^{r, \omega}\right)$ on $L_{\infty}\left(Q_{r, \omega}^{a},\left(\mathscr{B}_{t}\right)\right)$, and also on every $L_{p}\left(Q_{r, \omega}^{a}\right.$, $\left.\left(\mathscr{B}_{t}\right)\right)$ for $q_{0} \leq p<\infty$. These dynamic risk measures are time consistent for stopping times taking a finite number of values.

Proof There is a constant $C>0$ such that for all $Q_{r, \omega}^{a, a \mu} \in \tilde{\mathscr{Q}}_{r, \omega}(\Lambda),\|a \mu\|_{B M O\left(Q_{r, \omega}^{a}\right)} \leq$ $C$. It follows from the reverse Hölder inequality (19), that for all non negative measurable $Y, E_{Q_{r, \omega}^{a, a \mu}}\left(Y \mid \mathscr{B}_{s}\right) \leq K_{C}\left(E_{Q_{r, \omega}^{a}}\left(|| Y||^{q_{0}} \mid \mathscr{B}_{s}\right)^{\frac{1}{q_{0}}}\right.$. Thus $Y \rightarrow E_{Q_{r, \omega}^{a, a \mu}}\left(Y \mid \mathscr{B}_{s}\right)$ defines a linear continuous map on $L_{p}\left(Q_{r, \omega}^{a}\right)$ with values $L_{p}\left(Q_{r, \omega}^{a}\right)$ for all $q_{0} \leq p \leq \infty$, and that for given $p$, the norms of these linear maps are uniformly bounded for $\Lambda$ valued. From Proposition 3, it follows then that Eq. (25) defines a dynamic risk measure $\left(\rho_{s, t}^{r, \omega}\right)$ on $L_{p}\left(Q_{r, \omega}^{a},\left(\mathscr{B}_{t}\right)\right)$ for all $q_{0} \leq p<\infty$ in case (GC1). Under assumption (GC2), equation (25) defines a dynamic risk measure $\left(\rho_{s, t}^{r, \omega}\right)$ on $L_{p}\left(Q_{r, \omega}^{a},\left(\mathscr{B}_{t}\right)\right)$ for all $L_{p}, q_{0} \leq p \leq \infty$. 
The time consistency for stopping times taking a finite number of values follows from the stability property of the set of probability measures as well as the cocycle and local property of the penalties (cf. [5] in $L_{\infty}$ case). The proof is the same in $L_{p}$ case.

\section{Strong Feller Property}

\subsection{Feller Property for Continuous Parameters}

We assume that the progressively measurable function $g$ is a Caratheodory function on $I R_{+} \times \Omega \times \mathbb{R}^{n}$, that is for all $u,(\omega, x) \rightarrow g(u, \omega, x)$ is continuous. The support of $Q_{r, \omega}^{a, \mu}$ is contained in the paths $\omega^{\prime}$ continuous on $[r, \infty[$ (Proposition 1) and which coincide with $\omega$ on $[0, r]$. It follows that for every function $\lambda$ progressively continuous bounded, and all $u>r$, the function $\omega^{\prime} \rightarrow g\left(u, \omega^{\prime}, \lambda\left(u, \omega^{\prime}\right)\right)$ is continuous on the support of $Q_{r, \omega}^{a, a \lambda}$. We prove then the following Feller property for the penalty.

Proposition 5 Let a be progressively continuous bounded such that a $(s, \omega)$ is invertible for all $(s, \omega)$. Let $\lambda$ be progressively continuous bounded. Assume that $g$ is a real valued Caratheodory function satisfying the growth condition (GC1) or (GC2).

1. There is a strictly progressive real valued map $L(g)$ on $[0, t] \times \Omega \times \mathbb{R}^{n}$ continuous on $\left\{(s, \omega, x), \omega=\omega *_{s} x\right\}$ such that

$$
E_{Q_{s, \omega *}^{a, a \lambda} x} \int_{s}^{t} g\left(u, \omega^{\prime}, \lambda\left(u, \omega^{\prime}\right) d u\right)=L(g)(s, \omega, x) \quad \forall s \leq t \quad \omega \text { and } x
$$

2. For all $0 \leq r \leq s \leq t$, and $\omega \in \Omega$, there is a $Q_{r, \omega}^{a, \lambda}$-null set $N$ such that for all $\omega_{1} \in N^{c}$,

$$
E_{Q_{r, \omega}^{a, a \lambda}}\left(\int_{s}^{t} g\left(u, \omega^{\prime}, \lambda\left(u, \omega^{\prime}\right)\right) d u \mid \mathscr{B}_{S}\right)\left(\omega_{1}\right)=L(g)\left(s, \omega_{1}, X_{S}\left(\omega_{1}\right)\right)
$$

Proof We only need to prove 1.

Step 1: Assume that the function $g$ is bounded. Then $\int_{s}^{t} g\left(u, \omega^{\prime}, \lambda\left(u, \omega^{\prime}\right)\right) d u$ is a continuous bounded function of $\omega^{\prime}$ on the support of $Q_{s, \omega}^{a, a \lambda}$. The map $\lambda$ being progressively continuous bounded, the continuity property for $L(g)$ follows easily from Theorem 1.

Step 2: general case. Notice that $\lambda$ is bounded.

Thus under assumption (GC2), $g\left(u, \omega^{\prime}, \lambda\left(u, \omega^{\prime}\right)\right.$ is uniformly bounded and the continuity property of $L(g)$ follows from step 1 . 
Under assumption (GC1), let $\left(s_{n}, \omega_{n}, x_{n}\right), \omega_{n}=\omega_{n} *_{s_{n}} x_{n}$ with limit $(s, \omega, x), \omega=$ $\omega *_{s} x$. By definition the sequence $\omega_{n} *_{s_{n}} x_{n}$ has limit $\omega *_{s} x$. It follows from [3] that the set of probability measures $\mathscr{Q}=\left\{Q_{s_{n}, \omega_{n} *_{s_{n}} x_{n}}^{a \lambda}, n \in N\right\} \cup\left\{Q_{s, \omega *_{s} x}^{a \lambda}\right\}$ is weakly relatively compact and thus tight. Thus for all $\eta>0$, there is a compact set $\mathscr{K}$ such that $Q\left(\mathscr{K}^{c}\right)<\eta$ for all $Q \in \mathscr{Q}$. From the growth condition (GC1), the existence of a uniform bound for $E_{Q}\left[\int_{s}^{t}\left(1+\sup _{s \leq u}\left\|X_{u}\right\|^{m}\right) d u\right]^{k}$ for $Q \in \mathscr{Q}$ and the Hölder inequality, it follows that there is a progressively continuous bounded function $g_{1}$ such that for all $Q$ in $\mathscr{Q}$,

$$
E_{Q} \int_{s}^{t}\left(\| g\left(u, \omega,(a \lambda)(u, \omega)-g_{1}(u, \omega) \|\right)\right) d u \leq \varepsilon .
$$

The result follows then from step 1 .

We introduce now a class of $\mathscr{B}_{t}$ measurable functions on $\Omega$ satisfying a continuity condition derived from the progressive continuity condition that we have introduced for progressive functions and from the continuity property proved in Theorem 1 .

Definition 16 Let $t>0$. The function $h$ defined on $\Omega$ belongs to $\mathscr{C}_{t}$ if there is a function $\tilde{h}$ on $\Omega \times \mathbb{I R}^{n}$ such that

- $h(\omega)=\tilde{h}\left(\omega *_{t} X_{t}(\omega), X_{t}(\omega)\right)$

- $\tilde{h}(\omega, x)=\tilde{h}\left(\omega^{\prime}, x\right)$ if $\omega(u)=\omega^{\prime}(u) \forall u<t$

and such that $\tilde{h}$ is continuous bounded on $\left\{(\omega, x), \omega=\omega *_{t} x\right\} \subset \Omega \times \mathbb{R}^{n}$

Corollary 3 Assume that a is progressively continuous bounded and that a $(s, \omega)$ is invertible for all $(s, \omega)$. Let $\lambda$ be progressively continuous bounded. Let $h \in \mathscr{C}_{t}$. Asssume that the penalty $\alpha_{s, t}$ is given by Eq.(20) for some Caratheodory function $g$ on $\mathbb{I}_{+} \times \Omega \times \mathbb{R}^{n}$ satifying the growth condition (GC1) or (GC2). There is a strictly progressive map $L^{a, \lambda}(h)$ continuous on $\left\{\left(u, \omega *_{u} x, x\right), 0 \leq u \leq t\right\}$ such that for all $0 \leq r_{0} \leq r \leq t$,

$$
\begin{aligned}
L^{a, \lambda}(h)(t, \omega, x) & =\tilde{h}(\omega, x) \\
L^{a, \lambda}(h)(r, \omega, x) & =E_{Q_{r, \omega * r x}^{a, a \lambda}}(h)-\alpha_{r, t}\left(Q_{r, \omega *_{r} x}^{a, a \lambda}\right) \\
& =\left[E_{Q_{r_{0}, \omega_{0}}^{a, a \lambda}}\left(h \mid \mathscr{B}_{r}\right)-\alpha_{r, t}\left(Q_{r_{0}, \omega_{0}}^{a, a \lambda}\right)\right]\left(\omega *_{r} x\right) Q_{r_{0}, \omega_{0}}^{a, a \lambda} \text { a.s. }
\end{aligned}
$$

Proof The result follows from Theorem 1 and from Proposition 5.

\subsection{Feller Property for the Dynamic Risk Measure}

Proposition 6 Let $\mu$ in $\tilde{L}(\Lambda)$ (Definition 11). Let $t>0$ and $h \in \mathscr{C}_{t}$. Asssume that the penalty $\alpha_{s, t}$ is given by Eq.(20) for some Caratheodory function $g$ on $\mathbb{R}_{+} \times \Omega \times \mathbb{R}^{n}$ satifying the growth condition (GC1) or (GC2). There is a strictly progressive map 
$\Phi_{\mu}(h)$ continuous on $\left\{(u, \omega, x), \omega=\omega *_{u} x, u \leq t\right\}$ such that $\Phi_{\mu}(h)(t, \omega, x)=$ $\tilde{h}(\omega, x)$ for all $\omega$, and such that for all $r \leq s \leq t$, there is a process $v_{s}$ in $\tilde{L}(\Lambda)$ such that

$$
\begin{gathered}
\Phi_{\mu}(h)(s, \omega, x)=E_{Q_{s, \omega * s}^{a, a v_{s} x}}(h)-\alpha_{s, t}\left(Q_{s, \omega *_{s} x}^{a, a v_{s}}\right) \\
E_{Q_{r, \omega_{0}}^{a, a v_{s}}}\left(h \mid \mathscr{B}_{s}\right)\left(\omega^{\prime}\right)-\alpha_{s, t}\left(Q_{r, \omega_{0}}^{a, a v_{s}}\right)\left(\omega^{\prime}\right)=\Phi_{\mu}(h)\left(s, \omega^{\prime}, X_{s}\left(\omega^{\prime}\right)\right) \quad Q_{r, \omega_{0}}^{a} \text { a.s. }
\end{gathered}
$$

and

$$
E_{Q_{r, \omega_{0}}^{a, a \mu}}\left(h \mid \mathscr{B}_{S}\right)-\alpha_{s, t}\left(Q_{r, \omega_{0}}^{a, a \mu}\right) \leq E_{Q_{r, \omega_{0}}^{a, a v_{s}}}\left(h \mid \mathscr{B}_{S}\right)-\alpha_{s, t}\left(Q_{r, \omega_{0}}^{a, a v_{s}}\right) Q_{r, \omega_{0}}^{a} \text { a.s. }
$$

Proof The proof is done in two steps. The first step is the construction of $\Phi_{\mu}(h)$ given $\mu$. The second step is the construction of $v_{s}$ given $\mu$ and $s$. $\mu$ belongs to $\tilde{L}(\Lambda)$. From Definition 11 , let $0=s_{0}<\cdot .<s_{i}<s_{i+1} \cdots<s_{k}<s_{k+1}=\infty$ be a finite partition such that Eq. (13) is satisfied.

- First step: construction of $\Phi_{\mu}(h)$. We construct $\Phi_{\mu}(h)$ recursively on $\left[s_{i}, s_{i+1}[\right.$.

Let $s_{n}$ be such that $s_{n}<t \leq s_{n+1}$. From Corollary 3, for all $j \in I_{n}$ there is a strictly progressive map $L^{a, \lambda_{n, j}}(h)$ continuous on $\left\{(u, \omega, x), \omega=\omega *_{u} x, u \leq t\right\}$ satisfying Eq. (29). Let

$$
\Phi_{\mu}(h)(s, \omega, x)=\sup _{j \in I_{n}} L^{a, \lambda_{n, j}}(h)(s, \omega, x) \quad \forall s \in\left[s_{n}, t[\right.
$$

Let $h_{n}(\omega)=\Phi_{\mu}(h)\left(s_{n}, \omega, X_{s_{n}}(\omega)\right)$. The function $h_{n}$ belongs to $\mathscr{C}_{s_{n}}$ and $\tilde{h}_{n}(\omega, x)=\Phi_{\mu}(h)\left(s_{n}, \omega, x\right)$. Then we can proceed on $\left[s_{n-1}, s_{n}\right.$ [ with $h_{n}$. We construct recursively the strictly progressive map $\Phi_{\mu}(h)$ continuous on $\{(u, \omega, x), \omega=$ $\left.\omega *_{u} x, u \leq t\right\}$. Notice that for all $s \in\left[s_{i}, s_{i+1}\right]$, there are $\mathscr{B}_{s}$ measurable sets $\left(C_{s, j}\right)_{j \in I_{i}}$, such that

$$
\Phi_{\mu}(h)(s, \omega, x)=\sum_{j \in I_{i}} 1_{C_{s, j}}(\omega) L^{a, \lambda_{i, j}}\left(h_{i+1}\right)(s, \omega, x)
$$

- Second step: Given $s \in[r, t]$, construction of the process $v_{s}$.

There is a unique $k$ such that $\left.s \in] s_{k}, s_{k+1}\right]$. For $i>k$ for all $\left.\left.u \in\right] s_{i}, s_{i+1}\right]$, define

$$
v_{s}(u, \omega)=\sum_{j \in I_{i}} 1_{C_{s_{i}, j}}(\omega) \lambda_{i, j}(u, \omega)
$$

And for $\left.u \in] s, s_{k+1}\right]$, define

$$
v_{s}(u, \omega)=\sum_{j \in I_{k}} 1_{C_{s, j}}(\omega) \lambda_{k, j}(u, \omega)
$$


and $v_{s}(u, \omega)=\mu(u, \omega)$ for all $0 \leq u \leq s$. It follows from the construction of $v_{s}$ that the process $v_{s}$ belongs to $\tilde{L}(\Lambda)$. It follows also recursively that for all $i>k$ and $\omega \in \Omega$,

$$
E_{Q_{s_{i}, \omega}^{a, a v_{s}}}(h)-\alpha_{s_{i}, t}\left(Q_{s_{i}, \omega}^{a, a v_{s}}\right)=\Phi_{\mu}(h)\left(s_{i}, \omega, X_{s_{i}}(\omega)\right)
$$

and for all $\omega \in \Omega$,

$$
E_{Q_{s, \omega}^{a, a v_{s}}}(h)-\alpha_{s, t}\left(Q_{s, \omega}^{a, a v_{s}}\right)=\Phi_{\mu}(h)\left(s, \omega, X_{s}(\omega)\right)
$$

By construction the following inequality is satisfied:

$$
E_{Q_{s, \omega}^{a, a \mu}}(h)-\alpha_{s, t}\left(Q_{s, \omega}^{a, a \mu}\right) \leq \Phi_{\mu}(h)\left(s, \omega, X_{s}(\omega)\right)
$$

It follows then from Proposition 2 that

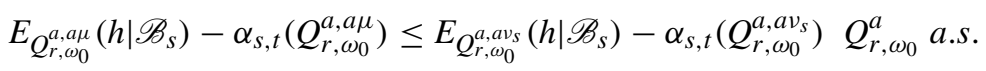

and

$$
\left[E_{Q_{r, \omega_{0}}^{a, a \nu_{s}}}\left(h \mid \mathscr{B}_{s}\right)-\alpha_{s, t}\left(Q_{r, \omega_{0}}^{a, a v_{s}}\right)\right]\left(\omega^{\prime}\right)=\Phi_{\mu}(h)\left(s, \omega^{\prime}, \omega^{\prime}(s)\right) Q_{r, \omega_{0}}^{a} \text { a.s. }
$$

Theorem 4 Assume that the hypothesis of Theorem 3 are satisfied and that $g$ is Caratheodary function. The time consistent dynamic risk measure $\left(\rho_{s, t}^{r, \omega}\right)_{r \leq s \leq t}$ defined on $L^{\infty}\left(\Omega, \mathscr{B}, Q_{r, \omega}^{a}\right)$ by Eq.(25) satisfies the following Feller property: For every function $h \in \mathscr{C}_{t}$, there is a progressive map $R(h)$ on $R_{+} \times \Omega, R(h)(t, \omega)=h(\omega)$, such that $\bar{R}(h)$ is lower semi continuous on $\left\{(u, \omega, x), u \leq t, \omega=\omega *_{u} x\right\}$ and such that the following equation is satisfied

$$
\begin{gathered}
\forall s \in[r, t], \forall \omega^{\prime} \in \Omega, \rho_{s, t}^{s, \omega^{\prime}}(h)=R(h)\left(s, \omega^{\prime}\right) \\
\forall 0 \leq r \leq s \leq t, \quad \rho_{s, t}^{r, \omega}(h)\left(\omega^{\prime}\right)=\bar{R}(h)\left(s, \omega^{\prime}, \omega^{\prime}(s)\right) Q_{r, \omega}^{a} \text { a.s. }
\end{gathered}
$$

$\left(\bar{R}(h)\right.$ denotes the strictly progressive map on $\mathbb{R}_{+} \times \Omega \times \mathbb{R}^{n}$ associated to $R(h)$ in the one to one corrrespondence introduced in Sect. 2).

Proof For all $\mu \in \tilde{L}(\Lambda)$, let $\Phi_{\mu}(h)$ be the strictly progressive map, continuous on $\left\{(u, \omega, x), \omega=\omega *_{u} x, u \leq t\right\}$ constructed in Proposition 6. Let $\bar{R}(h)=$ $\sup _{\mu \in \tilde{L}(\Lambda)} \Phi_{\mu}(h)$. The function $\bar{R}(h)$ is then lower semi continuous on $\{(u, \omega, x), \omega=$ $\left.\omega *_{u} x, u \leq t\right\}$. Let $R(h)(u, \omega)=\bar{R}(h)\left(u, \omega, X_{u}(\omega)\right)$. Equations (40) and (41) follow easily from Proposition 6. 


\section{Existence of Viscosity Solutions for Path-dependent PDEs}

\subsection{Existence of Viscosity Supersolutions}

Recall that $\Lambda$ is a closed convex lower hemicontinuous multivalued mapping from $X$ into $\mathbb{R}^{n}$ (Sect.4.2). Let $f$ be the convex conjugate of $g$ defined as

$$
f(u, \omega, z)=\sup _{y \in \Lambda(u, \omega)}\left(z^{*} y-g(u, \omega, y)\right)
$$

We prove now that for all $h \in \mathscr{C}_{t}$ the map $R(h)$ of Theorem 4 leads to viscosity solutions for the following semi-linear second order PDE.

$$
\left\{\begin{aligned}
-\partial_{u} v(u, \omega)-\mathscr{L} v(u, \omega)-f\left(u, \omega, a(u, \omega) D_{x} v(u, \omega)\right) & =0 \\
v(t, \omega) & =f(\omega) \\
\mathscr{L} v(u, \omega)=\frac{1}{2} \operatorname{Tr}\left(a(u, \omega) D_{x}^{2}(v)(u, \omega)\right) &
\end{aligned}\right.
$$

Theorem 5 Fix $\left(t_{0}, \omega_{0}\right)$. Assume that the mutivalued set $\Lambda$ is $B M O\left(Q_{t_{0}, \omega_{0}}^{a}\right)($ Definition 15). Assume that the function $g$ satisfies the preceding hypothesis ( $g$ is a Caratheodory function and satisfies the growth condition (GC1) or (GC2)). Assume furthermore that $g$ is upper semicontinuous on $\{(s, \omega, y),(s, \omega) \in X, y \in$ $\Lambda(s, \omega, \omega(s)\}$. For all $r$ and $\omega$, let $\left(\rho_{s, t}^{r, \omega}\right)$ be the dynamic risk measure given by Eq. (24) where the penalty satisfies Eq.(20).

Let $h \in \mathscr{C}_{t}$. The function $R(h)$ is progressive and $\bar{R}(h)$ is lower semi continuous on $\left\{(u, \omega, x), \omega=\omega *_{u} x, u \leq t\right\}$ (Theorem 4$) . R(h)$ is a viscosity supersolution of the path-dependent second order partial differential equation (43) at each point $\left(t_{0}, \omega_{0}\right)$ such that $f\left(t_{0}, \omega_{0}, a\left(t_{0}, \omega_{0}\right) z\right)$ is finite for all $z$.

Proof Let $x_{0}=\omega_{0}\left(t_{0}\right)$. From Theorem 4, the function $R(h)$ is progressive and $\bar{R}(h)$ is lower semi continuous on $\left\{(u, \omega, x), \omega=\omega *_{u} x, u \leq t\right\}$. We prove first that $R(h)$ is bounded from below on some $D_{\varepsilon}\left(t_{0}, \omega_{0}\right) . R(h)(u, \omega) \geq E_{Q_{u, \omega}^{a}}(h)-\alpha_{u t}\left(Q_{u, \omega}^{a}\right)$. For all given $\left.k \geq 2, E_{Q_{u, \omega}^{a}}\left(\sup _{s \leq u \leq t}\left(1+\left\|X_{t}\right\|\right)^{k}\right)\right)$ is uniformly bounded for $(u, \omega) \in$ $D_{\varepsilon}\left(t_{0}, \omega_{0}\right)$. The result follows then from either the (GC1) condition or the (GC2).

Let $\phi$ progressive, $\bar{\phi}$ in $\mathscr{C}_{b}^{1,0,2}\left(\mathbb{R}_{+} \times \Omega \times \mathbb{R}^{n}\right)$ such that $\phi\left(t_{0}, \omega_{0}\right)=R(h)\left(t_{0}, \omega_{0}\right)$ and $\left(t_{0}, \omega_{0}\right)$ is a local minimizer of $R(h)-\phi$ on $D_{\eta}\left(t_{0}, \omega_{0}\right)$ for some $\eta>0$.

- Step 1: Continuity properties

By hypothesis $f\left(t_{0}, \omega_{0}, a\left(t_{0}, \omega_{0}\right) D_{x} \bar{\phi}\left(t_{0}, \omega_{0}, x_{0}\right)\right)<\infty$. Thus for all $\varepsilon>0$, there is $\lambda_{0} \in \Lambda\left(t_{0}, \omega_{0}\right)$ such that

$$
D_{x} \bar{\phi}\left(t_{0}, \omega_{0}, x_{0}\right)^{t} a\left(t_{0}, \omega_{0}\right) \lambda_{0}-g\left(t_{0}, \omega_{0}, \lambda_{0}\right)>f\left(t_{0}, \omega_{0}, a\left(t_{0}, \omega_{0}\right) D_{x} \bar{\phi}\left(t_{0}, \omega_{0}, x_{0}\right)\right)-\varepsilon
$$

The multivalued set $\Lambda$ is assumed to be lower hemicontinuous. It follows that for all $K>0, \Lambda_{K}$ is also lower hemicontinuous, where $\Lambda_{K}(u, \omega, x)=\{z \in$ 
$\Lambda(u, \omega, x),\|z\|<K\}$. Choose $K>\left\|\lambda_{0}\right\|$. From Theorem 2 , there is thus a continuous bounded selector $\bar{\lambda}(u, \omega, x)$ of $\Lambda_{K}$ defined on $X$ such that $\bar{\lambda}\left(t_{0}, \omega_{0}, x_{0}\right)=\lambda_{0}$. From the upper semi-continuity condition satisfied by $g$, and the continuity of the map $\bar{\lambda}$, it follows that for all $\varepsilon>0$, there is $\eta_{1}>0$, such that for $t_{0} \leq u \leq t \leq$ $t_{0}+\eta_{1}, d\left(\omega, \omega_{0}\right)<\eta_{1}$ and $\left\|\omega(t)-x_{0}\right\|<\eta_{1}$,

$$
g(t, \omega, \bar{\lambda}(t, \omega, \omega(t)))-g\left(t_{0}, \omega_{0}, \lambda_{0}\right)<\varepsilon
$$

From the continuity of the function $\bar{\lambda}$, the hypothesis $\bar{\phi} \in \mathscr{C}_{b}^{1,0,2}$ and the progressive continuity of $a$, there is $\eta_{2}$ such that for $t_{0} \leq u \leq t \leq t_{0}+\eta_{2},\left\|x-x_{0}\right\|<\eta_{2}$ and $d\left(\omega *_{u} x, \omega_{0}\right)<\eta_{2}$,

$$
\begin{array}{r}
\mid \partial_{u} \bar{\phi}(u, \omega, x)+\frac{1}{2} \operatorname{Trace}\left(D_{x}^{2} \bar{\phi} \bar{a}(u, \omega, x)\right)+\left(D_{x} \bar{\phi}^{t} \bar{a} \bar{\lambda}\right)(u, \omega, x)- \\
\left.\partial_{u} \bar{\phi}\left(t_{0}, \omega_{0}, x_{0}\right)+\frac{1}{2} \operatorname{Trace}\left(D_{x}^{2} \bar{\phi} \bar{a}\right)\left(t_{0}, \omega_{0}, x_{0}\right)\right)+D_{x} \bar{\phi}^{t} \bar{a}\left(t_{0}, \omega_{0}, x_{0}\right) \lambda_{0} \mid \leq \varepsilon
\end{array}
$$

The maps $a$ and $\lambda$ are bounded. It follows from [22] that there is $0<\alpha<$ $\inf \left(\eta, \eta_{1}, \eta_{2}\right)$ such that

$$
Q_{t_{0}, \omega_{0}}^{a, a \lambda}(A)<\varepsilon \text { with } A=\left\{\omega \mid \sup _{t_{0} \leq u \leq t_{0}+\alpha}\left\|\omega(u)-\omega_{0}\left(t_{0}\right)\right\| \geq \inf \left(\eta, \eta_{1}, \eta_{2}\right)\right\}
$$

Let $C=\left\{\omega, \omega(u)=\omega_{0}(u) \forall 0 \leq u \leq t_{0}, \quad \sup _{t_{0} \leq u \leq t_{0}+\alpha}\left\|\omega(u)-\omega_{0}\left(t_{0}\right)\right\|<\right.$ $\left.\inf \left(\eta, \eta_{1}, \eta_{2}\right)\right\}$

- Step 2: Time consistency

For all $0<\beta<\alpha$, let $\delta$ be the stopping time $\delta=\beta 1_{C}$. The stopping time $\delta$ takes only 2 different values. By definition of the probability measure $Q_{t_{0}, \omega_{0}}^{a, a \lambda}$, it follows from (47) that $Q_{t_{0}, \omega_{0}}^{a, a \lambda}(C)>(1-\varepsilon)$.

The dynamic risk measure $\left(\rho_{u, v}^{t_{0}, \omega_{0}}\right)_{0 \leq u \leq v}$, is time consistent for stopping times taking a finite number of values, thus

$$
\rho_{t_{0}, t}^{t_{0}, \omega_{0}}(h)=\rho_{t_{0}, t_{0}+\delta}^{t_{0}, \omega_{0}}\left(\rho_{t_{0}+\delta, t}^{t_{0}, \omega_{0}}(h)\right)
$$

From Theorem 4, the lower semi continuous function $\bar{R}(h)$ satisfies: $\left(\rho_{t_{0}+\delta, t}^{t_{0}, \omega_{0}}\right.$ $(h)(\omega)=\bar{R}(h)\left(t_{0}+\delta, \omega, X_{t_{0}+\delta}(\omega)\right) Q_{t_{0}, \omega_{0}}^{a}$ a.s., and $\rho_{t_{0}, t}^{t_{0}, \omega_{0}}(h)=R(h)\left(t_{0}, \omega_{0}\right)$. Let $\bar{\lambda}$ be the continuous function defined in step 1 . By hypothesis $R(h) \geq \phi$ on $D_{\eta}\left(t_{0}, \omega_{0}\right)$. For all $\omega \in C$ and $u \leq \beta,\left(u, \omega *_{u} \omega(u)\right) \in D_{\eta}\left(t_{0}, \omega_{0}\right)$. Since the functions $R(h)$ and $\phi$ are progressive it follows that $R(h) \geq \phi$ on $\left[t_{0}, t_{0}+\beta\right] \times C$. It follows then from the equality $\phi\left(t_{0}, \omega_{0}\right)=R(h)\left(t_{0}, \omega_{0}\right)$ and from the definition of $\rho_{t_{0}, t_{0}+\delta}^{t_{0}, \omega_{0}}$ that

$$
\phi\left(t_{0}, \omega_{0}\right) \geq E_{Q_{t_{0}, \omega_{0}}^{a, a \lambda}}\left[\bar{\phi}\left(t_{0}+\delta, \omega, X_{t_{0}+\delta}(\omega)\right)-\int_{t_{0}}^{t_{0}+\delta} g\left(u, \omega, \lambda\left(u, \omega, X_{u}(\omega)\right)\right) d u\right]
$$


- Step 3: Martingale problem

The probability measure $Q_{t_{0}, \omega_{0}}^{a, a \lambda}$ is solution to the martingale problem for $\mathscr{L}^{a, a \lambda}$ starting from $\omega_{0}$ at time $t_{0}$. The function $\bar{\phi}$ is strictly progressive and belongs to $\mathscr{C}_{b}^{1,0,2}\left(\mathbb{R}_{+} \times \Omega \times \mathbb{R}^{n}\right)$. It follows from the martingale property proved in [3] that

$$
\begin{aligned}
0 \geq & E_{Q_{t_{0}, \omega_{0}}^{a, a \lambda}}\left[\int_{t_{0}}^{t_{0}+\delta}\left(\partial_{u} \bar{\phi}\left(u, \omega, X_{u}(\omega)\right)+\frac{1}{2} \operatorname{Trace}\left(\left(D_{x}^{2} \bar{\phi} \bar{a}\right)\left(u, \omega, X_{u}(\omega)\right)\right)\right) d u\right] \\
& +E_{Q_{t_{0}, \omega_{0}}^{a, a \lambda}}\left[\int_{t_{0}}^{t_{0}+\delta}\left(D_{x} \bar{\phi}^{t} \bar{a} \bar{\lambda}\right)\left(u, \omega, X_{u}(\omega)\right)-g\left(u, \omega, \bar{\lambda}\left(u, \omega, X_{u}(\omega)\right)\right) d u\right]
\end{aligned}
$$

- Step 4: Conclusion

Divide (50) by $\beta$ and let $\beta$ tend to 0 . It follows from the definitions of $C$ and $\delta$ and the Eqs. (44)-(46) proved in step 1 and $Q_{t_{0}, \omega_{0}}^{a, a \lambda}(C)>1-\varepsilon$ that

$-\partial_{u} \bar{\phi}\left(t_{0}, \omega_{0}, x_{0}\right)-\mathscr{L} \bar{\phi}\left(t_{0}, \omega_{0}, x_{0}\right)-f\left(t_{0}, \omega_{0}, \sigma^{t}\left(t_{0}, \omega_{0}\right) D_{x} \bar{\phi}\left(t_{0}, \omega_{0}, x_{0}\right)\right) \geq-2 \varepsilon(1-\varepsilon)$

This gives the result.

\subsection{Existence of Viscosity Subsolutions}

In this section we will assume that the set $\Lambda$ has some uniform BMO property.

Definition 17 The multivalued mapping $\Lambda$ is uniformly BMO with respect to $a$ if there is a non negative progressively measurable map $\varphi$ and $C>0$ such that for all $0 \leq s$,

$$
\sup \{|\lambda|, \lambda \in \Lambda(s, \omega)\} \leq \varphi(s, \omega)
$$

and such that for all $\left(s^{\prime}, \omega^{\prime}\right)$, the unique solution to the martingale problem $\mathscr{L}^{a}$ starting from $\omega^{\prime}$ at time $s^{\prime}$ satisfies:

$$
Q_{s^{\prime}, \omega^{\prime}}^{a}\left(\int_{s^{\prime}}^{\infty} \varphi(s, \omega)^{2} d s\right) \leq C
$$

Of course the above condition is satisfied if $\sup _{\omega} \int_{0}^{\infty} \varphi(s, \omega)^{2} d s<\infty$.

The name "uniform BMO" property is justifed by the following result.

Lemma 2 Assume that the multivalued mapping $\Lambda$ is uniformly BMO with respect to $a$. Then for all $(r, \omega)$ and all process $\mu \Lambda$ valued such that $\bar{\mu}$ is $\mathscr{P} \times \mathscr{B}\left(\mathbb{R}^{n}\right)$ measurable, $\mu$ belongs to $B M O\left(Q_{r, \omega}^{a}\right)$ and $\|\mu\|_{B M O\left(Q_{r, \omega}^{a}\right)} \leq C$

Proof Let $r, \omega$ and a stopping time $\tau \geq r$. It follows from [22] and from uniqueness of the solution to the martingale problem for $\mathscr{L}^{a, 0}$ starting from $\omega^{\prime}$ at time $\tau\left(\omega^{\prime}\right)$ that 
for $Q_{r, \omega}^{a}$ almost all $\omega^{\prime}$,

$$
E_{Q_{r, \omega}^{a}}\left(\int_{\tau}^{\infty} \varphi(u, \omega)^{2} d u \mid \mathscr{B}_{\tau}\right)\left(\omega^{\prime}\right)=E_{Q_{\tau\left(\omega^{\prime}\right), \omega^{\prime}}^{a}}\left(\int_{\tau\left(\omega^{\prime}\right)}^{\infty} \varphi(u, \omega)^{2} d u\right) \leq C
$$

Let $h \in \mathscr{C}_{t}$. The function $\bar{R}(h)$ is lower semi continuous in viscosity sense but it is not necessarily upper semi continuous. Therefore we need to introduce the upper semi continuous envelope of $R(h)$ in the viscosity sense according to Sect. 2.3. Denote it $R(h)^{*}$.

$$
R(h)^{*}(s, \omega)=\limsup _{\eta \rightarrow 0}\left\{R(h)\left(s^{\prime}, \omega^{\prime}\right), \quad\left(s^{\prime}, \omega^{\prime}\right) \in D_{\eta}(s, \omega)\right\}
$$

Theorem 6 Let $\left(t_{0}, \omega_{0}\right)$ be given. Assume that the mutivalued set $\Lambda$ is uniformly $B M O$ with respect to $a$. Assume that the function $g$ is a Caratheodory function satisfying the growth condition (GC1) or (GC2) and that the Fenchel transform $f$ of $g$ is progressively continuous.

Let $h \in \mathscr{C}_{t}$. The map $R(h)^{*}$ is progressive, $\overline{R(h)^{*}}$ is upper semicontinuous in viscosity sense. $R(h)^{*}$ is a viscosity subsolution of the path-dependent second order partial differential equation (43).

Proof Let $x_{0}=\omega_{0}\left(t_{0}\right)$. The progressivity of $R(h)^{*}$ follows from the equality $D_{\eta}(s, \omega)=D_{\eta}\left(s, \omega *_{s} \omega(s)\right)$. The upper semicontinuity property follows from the definition of $R(h)^{*}$. We prove first that $R(h)$ is bounded on some $D_{\varepsilon}\left(t_{0}, \omega_{0}\right)$. $R(h)(u, \omega)=\sup _{\mu}\left(E_{Q_{u, \omega}^{a, a \mu}}(h)-\alpha_{u, t}\left(Q_{u, \omega}^{a, a \mu}\right)\right)$. For given $k \geq 2, E_{Q_{u, \omega}^{a}}\left(\sup _{s \leq u \leq t}(1+\right.$ $\left.\left.\left.\| X_{t}||\right)^{k}\right)\right)$ is uniformly bounded for $(u, \omega) \in D_{\varepsilon}$. The result follows then from either the (GC1) condition or the (GC2), and from the uniform BMO hypothesis with similar arguments as in the proof of Proposition 3.

Let $\phi$ progressive, $\bar{\phi} \in \mathscr{C}_{b}^{1,0,2}\left(\mathbb{R}_{+} \times \Omega \times \mathbb{R}^{n}\right)$ such that $\phi\left(t_{0}, \omega_{0}\right)=R(h)^{*}\left(t_{0}, \omega_{0}\right)$ and such that $\left(t_{0}, \omega_{0}\right)$ is a maximizer of $R(h)^{*}-\phi$ on $D_{\eta}\left(t_{0}, \omega_{0}\right)$.

- Step 1: Making use of the progressive continuity property of $a, f$ and of the regularity of $\bar{\phi}$, for all $n \in N^{*}$, there is $\eta_{n}>0, t_{0}+\eta_{n}<t$ such that for $t_{0} \leq u \leq t_{0}+\eta_{n}, d\left(\omega_{o}, \omega\right)<\eta_{n}$ and $\left\|x_{0}-\omega(u)\right\|<\eta_{n}$,

$$
f\left(u, \omega,\left(\bar{a} D_{x} \bar{\phi}\right)(u, \omega, \omega(u))\right) \leq f\left(t_{0}, \omega_{0},\left(\bar{a} D_{x} \bar{\phi}\right)\left(t_{0}, \omega_{0}, x_{0}\right)\right)+\frac{1}{n}
$$

$$
\begin{aligned}
& \mid \partial_{u} \bar{\phi}(u, \omega, \omega(u))+\left.\frac{1}{2} \operatorname{Trace}\left(D_{x}^{2} \bar{\phi} \bar{a}\right)(u, \omega, \omega(u))\right)- \\
&\left.\partial_{u} \bar{\phi}\left(t_{0}, \omega_{0}, x_{0}\right)+\frac{1}{2} \operatorname{Trace}\left(D_{x}^{2} \bar{\phi} \bar{a}\right)\left(t_{0}, \omega_{0}, x_{0}\right)\right) \mid \leq \frac{1}{n}
\end{aligned}
$$

Without loss of generality one can assume $\eta_{n}<\eta$.

The matrix valued process $a$ being bounded, it follows from [22] that there is $h_{n}$ such that for all $t_{0} \leq s \leq t_{0}+\eta$ and $\omega \in D_{\eta}\left(t_{0}, \omega_{0}\right)$, 


$$
Q_{s, \omega}^{a}\left(\left\{\omega^{\prime}, \sup _{s \leq u \leq s+h_{n}}\left\|\omega^{\prime}(u)-\omega(s)\right\|>\frac{\eta_{n}}{2}\right\}\right)<\varepsilon
$$

Without loss of generality one can assume that $h_{n}<\frac{\eta_{n}}{2}$.

For all $n>0$ choose $\left(t_{n}, \omega_{n}\right) \in D_{\frac{\eta_{n}}{2}}\left(t_{0}, \omega_{0}\right)$ such that $\lim _{n \rightarrow \infty} R(h)\left(t_{n}, \omega_{n}\right)=$ $R(h)^{*}\left(t_{0}, \omega_{0}\right)$ and

$$
\phi\left(t_{n}, \omega_{n}\right) \leq R(h)\left(t_{n}, \omega_{n}\right)+\frac{h_{n}}{n}
$$

Let $C_{n}=\left\{\omega^{\prime}, \sup _{t_{n} \leq u \leq t_{n}+h_{n}}\left\|\omega^{\prime}(u)-\omega_{n}\left(t_{n}\right)\right\| \leq \frac{\eta_{n}}{2}, \omega^{\prime}(v)=\omega_{n}(v), \forall v \leq t_{n}\right\}$. It follows from Eq. (55) that $Q_{t_{n}, \omega_{n}}^{a}\left(C_{n}\right)>1-\varepsilon$ for all $n$. From Lemma 2 there is a constant $C^{\prime}>0$ such that $\|a \mu\|_{B M O\left(Q_{t_{n}, \omega_{n}}^{a}\right)} \leq C^{\prime}$ for all $\mu \in \tilde{L}(\Lambda)$ and all $n$. From [15] it follows that there is $p_{0}$ such that the reverse Hölder inequality (19) is satisfied for all $\mathscr{E}(a \mu)$ and thus that for all $\mu \in \tilde{L}(\Lambda)$, and all $n, Q_{t_{n}, \omega_{n}}^{a, a \mu}\left(C_{n}^{c}\right) \leq K_{C}(\varepsilon)^{\frac{1}{q_{0}}}$ where $q_{0}$ is the conjugate exponent of $p_{0}$. The constants $q_{0}$ and $K_{c}$ depend neither on $\mu$ nor on $n$. Thus $\varepsilon$ can be chosen such that $Q_{t_{n}, \omega_{n}}^{a, a \mu}\left(C_{n}\right)>\frac{1}{2}$ for all $n$ and all $\mu \in \tilde{L}(\Lambda)$.

Let $\delta_{n}$ be the stopping time taking only two values $h_{n}$ and 0 defined by $\delta_{n}=h_{n} 1_{C_{n}}$.

- Step 2: Time consistency

Making use of the time consistency of the risk measure $\left(\rho_{u, v}^{t_{n}, \omega_{n}}\right)$, and of the following equations deduced from Theorem 4,

$$
\begin{gathered}
R(h)\left(t_{n}, \omega_{n}\right)=\rho_{t_{n}, t}^{t_{n}, \omega_{n}}(h) \\
\rho_{t_{n}+\delta_{n}, t}^{t_{n}, \omega_{n}}(h)=R(h)\left(t_{n}+\delta_{n}, .\right) Q_{t_{n}, \omega_{n}}^{a} \text { a.s. }
\end{gathered}
$$

it follows that

$$
R(h)\left(t_{n}, \omega_{n}\right)=\rho_{t_{n}, t_{n}+\delta_{n}}^{t_{n}, \omega_{n}}\left(R(h)\left(t_{n}+\delta_{n}, .\right)\right)
$$

It follows from the definition of the risk measure $\left(\rho_{t_{n}, t_{n}+\delta_{n}}^{t_{n}, \omega_{n}}\right)$, that for all $n$ there is a process $\mu_{n}$ in $\tilde{L}(\Lambda)$ such that

$$
R(h)\left(t_{n}, \omega_{n}\right) \leq E_{Q_{t_{n}, \omega_{n}}^{a, a \mu_{n}}}\left(R(h)\left(t_{n}+\delta_{n}, .\right)\right)-\alpha_{t_{n}, t_{n}+\delta_{n}}\left(Q_{t_{n}, \omega_{n}}^{a, a \mu_{n}}\right)+\frac{h_{n}}{n}
$$

For all $\omega^{\prime} \in C_{n},\left(t_{n}+h_{n}, \omega^{\prime} *_{t_{n}+h_{n}} \omega^{\prime}\left(t_{n}+h_{n}\right)\right) \in D_{\eta}\left(t_{0}, \omega_{0}\right)$. The functions $R(h)$ and $\phi$ are progressive and satisfy $R(h) \leq R(h)^{*} \leq \phi$ on $D_{\eta}\left(t_{0}, \omega_{0}\right)$. It follows that

$$
R(h)\left(t_{n}+\delta_{n}, \omega^{\prime}\right) \leq \phi\left(t_{n}+\delta_{n}, \omega^{\prime}\right) \quad \forall \omega^{\prime} \in C_{n}
$$

From Eqs. (56)-(58), it follows that

$$
\phi\left(t_{n}, \omega_{n}\right) \leq E_{Q_{t_{n}, \omega_{n}}^{a, a \mu_{n}}}\left[\phi\left(t_{n}+\delta_{n}, .\right)-\int_{t_{n}}^{t_{n}+\delta_{n}} g\left(u, \omega^{\prime}, \mu_{n}\left(u, \omega^{\prime}\right)\right) d u\right]+2 \frac{h_{n}}{n}
$$


- Step 3: Martingale problem

Given $\left(t_{n}, \omega_{n}\right)$, the probability measure $Q_{t_{n}, \omega_{n}}^{a, a \mu_{n}}$ is solution to the martingale problem $\mathscr{L}^{a, a \mu_{n}}$ starting from $\omega_{n}$ at time $t_{n}$. The strictly progressive function $\bar{\phi}$ belongs to $\mathscr{C}_{b}^{1,0,2}$. It follows from [3] and from Eq. (59) that

$$
\begin{aligned}
& 0 \leq E_{Q_{t_{n}, \omega_{n}}^{a, a \mu_{n}}}\left[\int_{t_{n}}^{t_{n}+\delta_{n}}\left(\partial_{u} \bar{\phi}\left(u, \omega^{\prime},\left(\omega^{\prime}(u)\right)\right)+\frac{1}{2} \operatorname{Trace}\left(D_{x}^{2} \bar{\phi} \bar{a}\right)\left(u, \omega^{\prime}, \omega^{\prime}(u)\right)\right) d u\right] \\
& +E_{Q_{t_{n}, \omega_{n}}^{a, a \mu_{n}}}\left[\int_{t_{n}}^{t_{n}+\delta_{n}}\left(D_{x} \bar{\phi}^{t}\left(u, \omega^{\prime}, \omega^{\prime}(u)\right) a\left(u, \omega^{\prime}\right) \mu_{n}\left(u, \omega^{\prime}\right)-g\left(u, \omega^{\prime}, \mu_{n}\left(u, \omega^{\prime}\right)\right)\right) d u\right]+2 \frac{h_{n}}{n}
\end{aligned}
$$

By definition of $f$ it follows that

$$
\begin{aligned}
0 \leq & E_{Q_{t_{n}, \omega_{n}}^{a, a \mu_{n}}}\left[\int_{t_{n}}^{t_{n}+\delta_{n}}\left(\partial_{u} \bar{\phi}\left(u, \omega^{\prime}, \omega^{\prime}(u)\right)\right)+\frac{1}{2} \operatorname{Trace}\left(\left(D_{x}^{2} \bar{\phi} \bar{a}\right)\left(u, \omega^{\prime}, \omega^{\prime}(u)\right)\right) d u\right] \\
& +E_{Q_{t_{n}, \omega_{n}}^{a, a \mu_{n}}}\left[\int_{t_{n}}^{t_{n}+\delta_{n}} f\left(u, \omega^{\prime},\left(\bar{a} D_{x} \bar{\phi}\right)\left(u, \omega^{\prime}, \omega^{\prime}(u)\right)\right) d u\right]+2 \frac{h_{n}}{n}
\end{aligned}
$$

- Step 4: Conclusion

Divide equation (61) by $h_{n}$ and let $n$ tend to $\infty$. The result follows from step 1, the inequality $Q_{t_{n}, \omega_{n}}^{a, a \mu_{n}}\left(C_{n}\right) \geq \frac{1}{2}$ for all $n$ and $\delta_{n}=h_{n} 1_{C_{n}}$.

\subsection{Existence of Viscosity Solutions on the Set of Continuous Paths}

On the set of continuous paths $\mathscr{C}\left(\mathbb{R}_{+} \times \mathbb{R}^{n}\right)$ we consider the uniform norm topology. In this section we assume that the function $a$ is only defined on $R_{+} \times \mathscr{C}\left(\mathbb{R}_{+} \times \mathbb{R}^{n}\right)$ and that it is continuous. For every continuous function $h$ on the space of continuous paths $\mathscr{C}\left(\mathbb{R}_{+} \times \mathbb{R}^{n}\right)$ such that $h(\omega)=h\left(\omega^{\prime}\right)$ if $\omega(u)=\omega^{\prime}(u)$ for all $u \leq t$, the corresponding function $R(h)$ is constructed as above. In this case the function $R(h)$ is defined only on the set of continuous paths (more precisely on $[0, t] \times \mathscr{C}\left(\mathbb{R}_{+} \times \mathbb{R}^{n}\right)$ ). We make use of the definition of viscosity solution on the set of continuous paths introduced in Sect. 2.4 (Definition 4). To prove that $R(h)$ is a viscosity supersolution, to define $R(h)^{*}$ and prove that it is a viscosity subsolution, we do not need to extend the functions $a$ nor $R(h)$. Indeed as the support of the probability measure $Q_{r, \omega}^{a}$ is contained in the set of continuous paths, we just need to use the restrictions of the function $\bar{\phi}$ and of its partial derivatives to the set $I R_{+} \times \mathscr{C}\left(\mathbb{R}_{+} \times I R^{n}\right) \times \mathbb{R} R^{n}$. The proofs given for Theorems 5 and 6 can be easily adapted to prove the analog result in the setting of continuous paths.

Thus the setting of continuous paths can be considered as a "particular case" of the setting of càdlàg paths. 


\section{Conclusion and Perspectives}

We have introduced new notions of regular solutions and viscosity solutions for pathdependent second order PDEs, Eq. (1), in the setting of càdlàg paths. In line with the recent literature on the topic, a solution of (1) must be searched among progressive functions, that is path-dependent functions depending at time $t$ on all the path up to time $t$. However, the notions of solutions introduced in the present paper differ from previous notions introduced in the literature on two major points:

- In contrast with other papers, we consider on the set $\Omega$ of càdlàg paths the Skorokhod topology. $\Omega$ is thus a Polish space. This property is fundamental for the construction of solutions for path-dependent PDE that we give in the present paper.

- The notions of partial derivative for progressive functions that we introduce are defined in a very natural way by considering a progressive function of two variables as a function of three variables.

In addition we introduce also a notion of viscosity solution on the set of continuous paths.

Making use of the martingale problem approach to path-dependent diffusion processes, we then construct time consistent dynamic risk measures $\rho_{s t}^{r, \omega}$. The stable set of probability measures used for the construction of $\rho_{s t}^{r, \omega}$ is a set generated by probability measures solution to the path-dependent martingale problem for $\mathscr{L}^{a, a \mu}$ starting form $\omega$ at time $r$. The path-dependent progressively continuous bounded function $a$ is given and takes values in the set of invertible non negative matrices. The path-dependent functions $\mu$ are progressively continuous and vary accordingly to a multivalued mapping $\Lambda$. This construction is done in a very general setting. In particular the coefficients $\mu$ are not uniformly bounded. We just assume that they satisfy some uniform BMO condition. To construct the penalties, we make use of a path-dependent function $g$ satisfying some polynomial growth condition with respect to the path and some $L_{2}$ condition with respect to the process $\mu$ related to the BMO condition. In contrast with the usual setting of BSDE, in all this construction no Lipschitz hypothesis are assumed. Notice however that the Lipschitz setting can also be studied within our approach: instead of starting with progressively continuous maps $a$ and $\mu$, one could start from a subfamily of maps which, for example, satisfy some uniform continuity condition (as $K$ Lipschitz maps).

We show that these risk measures provide explicit solutions for semi-linear pathdependent PDEs (2). First, we prove that the risk measures $\rho_{s t}^{r, \omega}$ satisfy the following Feller property. For every function $h \mathscr{B}_{t}$ measurable having some continuity property, there is a progressively lower semi-continuous function $R(h)$ such that $\rho_{r t}^{r, \omega}\left(h\left(X_{t}\right)\right)=$ $R(h)(r, \omega)$ and $R(h)(t, \omega)=h(t, \omega)$. Next, the function $R(h)$ is proved to be a viscosity supersolution for a semi-linear path-dependent PDE (2), where the function $f$ itself is associated to $g$ by duality on the multivalued mapping $\Lambda$. We prove also that the upper semi continuous envelope of $R(h)$ is a viscosity subsolution for the path-dependent semi linear second order PDE (2).

Here we have proved the progressive lower semi continuity for $R(h)$. To prove the progressive continuity property, additional hypothesis should be added, e.g. Lipschitz 
conditions. Another way of proving the continuity is to apply a comparison Theorem. The study of comparison theorems and of continuity properties in this setting, as well as the study of solutions to fully non linear path-dependent PDE will be the subject of future work.

Acknowledgments This work was partly done while at the Center for Advanced Studies at the Norwegian Academy of Science and Letters (CAS, Oslo). It is a pleasure to thank Giulia Di Nunno and Fred Espen Benth for the invitation to contribute to the CAS program in Stochastics in Environmental and Financial Economics (SEFE), and to give a talk at the inaugural SEFE conference.

Open Access This chapter is distributed under the terms of the Creative Commons Attribution Noncommercial License, which permits any noncommercial use, distribution, and reproduction in any medium, provided the original author(s) and source are credited.

\section{Appendix}

An important way of constructing time consistent dynamic risk measures is to construct a stable set of equivalent probability measures and to define on this set a penalty which is local and satisfies the cocycle condition [5]. Recall the following definitions

Definition 18 A set $\mathscr{Q}$ of equivalent probability measures on a filtered probability space $\left(\Omega, \mathscr{B},\left(\mathscr{B}_{t}\right)\right)$ is stable if it satisfies the two following properties:

1. Stability by composition

For all $s \geq 0$ for all $Q$ and $R$ in $\mathscr{Q}$, there is a probability measure $S$ in $\mathscr{Q}$ such that for all $X$ bounded $\mathscr{B}$-measurable,

$$
E_{S}(X)=E_{Q}\left(E_{R}\left(X \mid \mathscr{B}_{S}\right)\right)
$$

2. Stability by bifurcation

For all $s \geq 0$, for all $Q$ and $R$ in $\mathscr{Q}$, for all $A \in \mathscr{B}_{s}$, there is a probability measure $S$ in $\mathscr{Q}$ such that for all $X$ bounded $\mathscr{B}$-measurable,

$$
E_{S}\left(X \mid \mathscr{F}_{S}\right)=1_{A} E_{Q}\left(X \mid \mathscr{F}_{S}\right)+1_{A^{c}} E_{R}\left(X \mid \mathscr{F}_{S}\right)
$$

Definition 19 A penalty function $\alpha$ defined on a stable set $\mathscr{Q}$ of probability measures all equivalent is a family of maps $\left(\alpha_{s, t}\right), s \leq t$, defined on $\mathscr{Q}$ with values in the set of $\mathscr{B}_{s}$-measurable maps such that

(i) $\alpha$ is local:

For all $Q, R$ in $\mathscr{Q}$, for all $s$, for all $A$ in $\mathscr{B}_{s}$, the assertion $1_{A} E_{Q}\left(X \mid \mathscr{B}_{s}\right)=$ $1_{A} E_{R}\left(X \mid \mathscr{B}_{s}\right)$ for all $X$ bounded $\mathscr{B}_{t}$ measurable implies that $1_{A} \alpha_{s, t}(Q)=$ $1_{A} \alpha_{s, t}(R)$. 
(ii) $\alpha$ satisfies the cocycle condition: For all $r \leq s \leq t$, for all $Q$ in $\mathscr{Q}$,

$$
\alpha_{r, t}(Q)=\alpha_{r, s}(Q)+E_{Q}\left(\alpha_{s, t}(Q) \mid \mathscr{F}_{r}\right)
$$

Recall the following result from [5].

Proposition 7 Given a stable set $\mathscr{Q}$ of probability measures and a penalty $\left(\alpha_{s, t}\right)$ defined on $\mathscr{Q}$ satisfying the local property and the cocycle condition,

$$
\rho_{s t}(X)=\operatorname{essup}_{\mathrm{Q} \in \mathscr{Q}}\left(\mathrm{E}_{\mathrm{Q}}\left(\mathrm{X} \mid \mathscr{F}_{\mathrm{s}}\right)-\alpha_{\mathrm{st}}(\mathrm{Q})\right)
$$

defines a time consistent dynamic risk measure on $L_{\infty}\left(\Omega, \mathscr{B},\left(\mathscr{B}_{t}\right)\right)$ or on $L_{p}(\Omega, \mathscr{B}$, $\left.\left(\mathscr{B}_{t}\right)\right)$ if the corresponding integrability conditions are satisfied.

\section{References}

1. Aliprantis, C., Border, K.: Infinite Dimensional Analysis, 2nd edn. Springer, Berlin (1999)

2. Billingsley, P.: Convergence of Probability Measures. Wiley series in probability and mathematical statistics. Wiley, New York (1999)

3. Bion-Nadal, J.: Martingale problem for integral-differential operator with path dependent coefficient, preprint (2015)

4. Bion-Nadal, J.: Time consistent convex Feller processes and non linear second order partial differential equations, preprint. arXiv:1207.1742 (2012)

5. Bion-Nadal, J.: Dynamic risk measures: time consistency and risk measures from BMO martingales. Finance Stochast. 12, 219-244 (2008)

6. Bion-Nadal, J.: Time consistent dynamic risk processes. Stochast. Processes Appl. 119, 633654 (2008)

7. Cheredito, P., Delbaen, F., Kupper, M.: Dynamic monetary risk measures for bounded discrete time processes. Electron. J. Probab. 11, 57-106 (2008)

8. Cont, R., Fournié, D.: Functional Ito calculus and stochastic integral representation of martingales. Ann. Probab. 41, 109-133 (2013)

9. Delbaen, F.: The structure of $m$-stable sets and in particular of the set of risk neutral measures. Memoriam Paul-André Meyer, in: Lecture Notes in Mathematics 1874, 215-258 (2006)

10. Diffusion processes with continuous coefficients II. Commun. Pure Appl. Math. 22, 479-530 (1969)

11. Diffusion processes with continuous coefficients I. Commun. Pure Appl. Math. 22, 345-400 (1969)

12. Dupire, B.: Functional Ito calculus, papers.ssrn.com (2009)

13. Ekren I., Touzi N., Zhang J.: Viscosity solutions of fully nonlinear parabolic path dependent PDES. arXiv:1210.0006v3 (2014)

14. Ekren, I., Keller, C., Touzi, N., Zhang, J.: On viscosity solutions of path dependent PDES. Ann. Probab. 42(1), 204-236 (2014)

15. Kamazaki, N.: Continuous Exponential Martingales and BMO, lecture notes in mathematics 1579, Springer (1994)

16. Krylov, N.: Controlled Diffusion Processes. Springer (1980)

17. Pardoux, E., Peng, S.: Backward stochastic differential equations and quasi linear parabolic partial differential equations, lecture notes in CIS. Springer 176, 200-217 (1992)

18. Peng, S., Wang, F.: BSDE, path dependent PDE and non linear Feynman Kac formula, preprint. arXiv:1108.4317 (2011) 
19. Peng, S.: Backward stochastic differential equations nonlinear expectations and their applications, Proceedings of the International Congress of Mathematicians, Hyderabad, India 2010

20. Peng, S.: Note on viscosity solution of path dependent PDE and G Martingales, preprint. arXiv:1106.1144v2 (2012)

21. Peng, S.: Nonlinear expectations, nonlinear evaluations and risk measures. Lect. Notes Math. 1856, 165-253 (2004)

22. Strook, D.: Diffusion processes asociated with Levy generators. Z. Wahrscheinlichkeitstheorie verw. Gebiete 32, 209-244 (1975) 